\title{
A FORGOTTEN SOCIAL GROUP: A CHINESE PHOTOGRAPHIC ALBUM
}

\author{
by \\ Yanru Huang \\ Bachelor of Fine Arts, Cinema and Television \\ Hong Kong Baptist University, 2014
}

\author{
A Thesis Presented to Ryerson University \\ in partial fulfillment of the requirements of the degree of \\ Master of Arts \\ in the Program of
}

Film and Photography Preservation and Collections Management

Toronto, Ontario, Canada, 2017

(C) Yanru Huang 2017 
I hereby declare that I am the sole author of this thesis or dissertation.

I authorize Ryerson University to lend this thesis or dissertation to other institution or individuals for the purpose of scholarly research.

Yanru Huang

I further authorize Ryerson University to reproduce this thesis or dissertation by photocopying or by other means, in total or in part, at the request of other institution or individuals for the purpose of scholarly research.

Yanru Huang 
A Forgotten Social Group: A Chinese Photographic Album Master of Arts, 2017

Yanru Huang

Film and Photography Preservation and Collections Management Ryerson University

\section{Abstract}

This applied thesis project focuses on a Chinese photographic album Work. The Manufacturing Industry in Dongguan: Real-life Stories of Migrant Workers housed at the Archive of Modern Conflict in Toronto. It is a handmade photographic album by Chinese photographer Zhan Youbing, consisting of 55 objects including 50 digital color prints (D2T2 prints) featuring the life of Chinese migrant workers in Dongguan, the biggest labor market in Southern China. This thesis comprises four components: a literature review introducing the unique status of migrant workers within the historical context of China's urbanization and the preservation and conservation of photographic albums, an overview of the album, a complete illustrated catalogue of the 55 objects as well as a detailed instruction of the preservation of the album. 


\section{Acknowledgements}

I would like to take this opportunity to express my gratitude to all the individuals that offered me advice, encouragement and support for the completion of this thesis.

First, I would like to thank my first and second readers Marta Braun and Thierry Gervais for their guidance, patience and understanding.

At the AMC, I would like to thank Michelle Willison, David Franklin, Jennifer Graham, Stefanie Petrilli, and Parker Kay for their help and knowledge with which they generously shared throughout this project.

I would also like to thank all of my classmates and friends at Ryerson University, Raquel Moliterno, Madeleine Anne Bognar, Victoria Grace, Sasha Furlani, Locrin Stewart, Jeff Yu, Celio Barreto and Dee Psaila for always listening and giving me words of encouragement.

Lastly, I would like to thank my family and my partner Hua Huang for always believing in me and for their endless support and love. I love you all. 


\section{Dedication}

This thesis is dedicated to all the disadvantaged minorities in society. Please remember that you are not alone. Only love and faith can make impossibilities to possibilities. 


\section{Table of Contents}

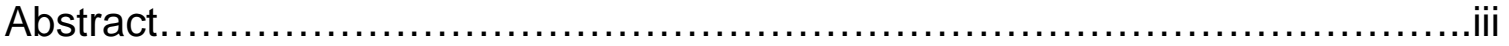

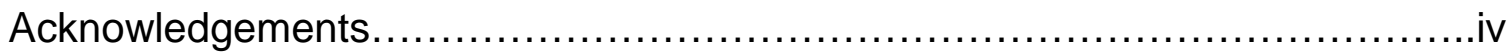

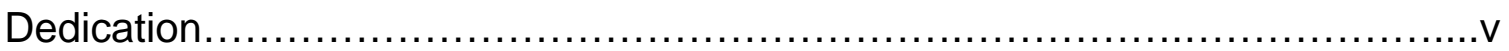

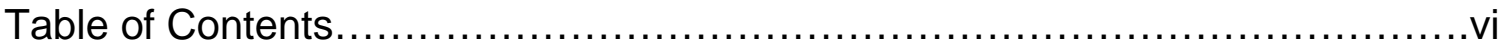

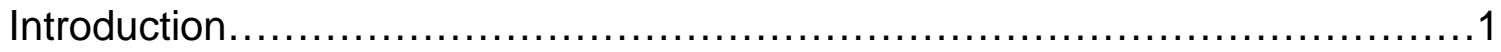

Chapter 1. Literature Review..........................................4

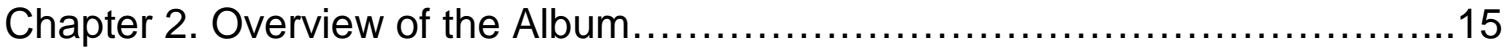

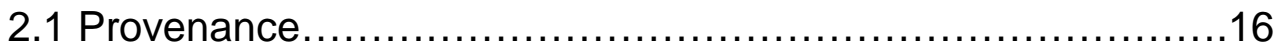

2.2 Exhibition History ........................................

Chapter 3. Catalogue of the Album

3.1 Translated Introduction of the Album ............................20

3.2 Introduction to the Catalogue................................

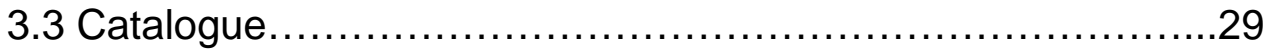

Chapter 4. Preservation/Housing Instruction

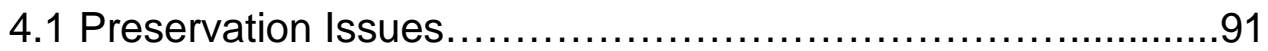

4.1.1 Condition of the Album .............................91

4.1.2 Test before Preservation...........................91

4.2 Making of the Box..........................................

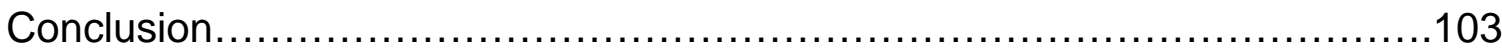

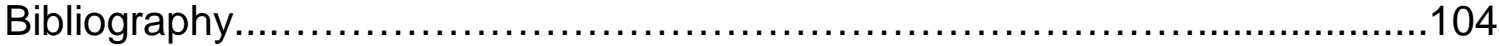




\section{Introduction}

Resulting from the open door policy and economic reforms in the 1980s, China has become "the world's labor factory" in the ongoing process of urbanization. It contributes not only to profound social changes in China but also a new working class comprised of 280 million Chinese migrant workers who drift to metropolises from rural areas trying to provide a better life for their families. Nowadays, "Made in China" products can be found in almost every corner of the world but little attention has been paid to those hands that created them. Chinese photographer Zhan Youbing is an exception. Zhan, starting as a migrant worker in 1995 in Dongguan, the biggest manufacturing city in Southern China, spent more than ten years working in factories, witnessing and understanding the struggles of migrant workers. Since 2006, he has taken over four hundred thousand photographs documenting the factory life of his fellow workers. ${ }^{1}$

The Archive of Modern Conflict (AMC) in Toronto has a photographic collection of thirty handmade photographic albums by Zhan. The subject of this applied thesis Work. The Manufacturing Industry in Dongguan: Real-Life Stories of Migrant Workers, by far the biggest and the most comprehensive album of Zhan's work, includes fifty photographs taken in Dongguan between 2008 and 2014. It also contains personal belongings of migrant workers collected by Zhan. Individually, each item is treated as a single object whose role is as important as

\footnotetext{
${ }^{1}$ Dingdang Liu, "Zhan Youbing: The Lens Are My Eyes," Siyuefeng.com, October 14, 2014, accessed May 06, 2017, http://zhanyoubing.siyuefeng.com/article/10047.
} 
that of the photographs in presenting the history of Chinese migrant workers. Together, they form a representative image of Chinese migrant workers, who work hard and live in obscurity.

This thesis is a practical project divided into four sections. The first section is a literature review that covers the unique social status of migrant workers within the historical context of China's urbanization. It also introduces literature on Chinese migrant workers describing the relationship between Chinese documentary photography and the growing attention drawn to Chinese migrant workers. Lastly, it reviews existing literature on the preservation of photographic albums and case studies of photographic albums in institutions. The second section provides an overview of the album, including its content, provenance, and exhibition history both in mainland China and overseas.

The third section addresses the practical component of the thesis. It begins with my translation of the introduction of the album, originally written in Chinese by Zhan Youbing. It covers a brief biography of Zhan describing his experience in becoming a photographer from a migrant worker, and his personal perspective on this social group. This section includes a complete illustrated catalogue of the album with a preface introducing the definition of each field of the catalogue. All the captions are translated into English in order to make the album more accessible to AMC's curatorial staff and future researchers. The fourth section analyzes the preservation issues of the album and provides an illustrated step- 
by-step instruction for constructing custom housing for the album. 


\section{Chapter 1. Literature Review}

Over the past 35 years, China has become the "world's factory" resulting from its open-door policy and economic reform. These changes brought about the rise of a new working class comprised of rural migrant workers. The maker of Work. The Manufacturing Industry in Dongguan: Real-Life Stories of Migrant Workers, Zhan Youbing, was one of them. Compared to the extensive attention given to China's thriving economy, the existence of this social group has been neglected for three decades. Now it has been gradually brought to the attention of different Chinese social classes and the rest of the world. The published writings discussed in this literature survey on Chinese migrant workers include books, critical essays, journals etc. However, studies on photography about Chinese migrant workers are relatively limited. The literature review is divided into three sections: the first discusses the formation of the new generation of migrant workers and their unique social status. The second focuses on Chinese documentary photography about migrant workers. The last section reviews the preservation of photographic albums, as well as various case studies of the conservation of albums in different institutions.

According to Han Changbing, Chinese Agriculture Secretary, migrant workers are divided into three generations. ${ }^{2}$ The first generation, who appeared in the 80 s, were farmers from the countryside who came to work in the cities. They

\footnotetext{
2 "Agriculture Secretary Han Changbin Talks About Post 1990s Migrant Workers," People.com.cn, February 1, 2010, accessed April 16, 2017, http://politics.people.com.cn/GB/1027/10893999.html.
} 
recognized themselves as both workers and peasants, drifting to the city while retaining their roots and souls in the hometown soil. Growing during the late 80s, the second generation, as Han puts it, has become the main force of migrant workers. And those born in the early 1990s are referred to as the third generation, namely the new generation of migrant workers. In Han's definition, the ample scale of the second and the third generation constitutes the main body of modern migrant workers. Han points out the inherent characteristics of the new generation of migrant workers. Different from the previous generations, their emotional attachment towards soil and agriculture diminished greatly once they adjusted to the life style of modern cities. Eager to improve their life, they worked hard to fight for a place in the city. Gradually, they became obsessed with the favorable conditions of urban living and resisted any return to the rural regions. When exposed to certain prejudice and inequality, the new generation of migrant workers demanded the same equal rights as those of urban citizens.

Wang Chunguang, a researcher at the Chinese Academy of Social Sciences, began his studies on the floating population of the third generation of migrant workers in 2001. In The Social Identity of the New Generation of Migrant Workers and Relationship of Urban and Rural Integration (2001), Wang asserts that an intergenerational difference has occurred. ${ }^{3}$ Unlike their parents, migrants of the new generation are granted greater access to higher levels of education and more career opportunities, which to some degree have become their primary

\footnotetext{
${ }^{3}$ Chunguang Wang, "The Social Identity of the New Generation of Migrant Workers and Relationship of Urban and Rural Integration," Sociological Studies 16, no. 3 (2001): 63-76.
} 
motive to work in the cities. He also concludes that the social identity of the new generation tends to become more and more indefinite than that of the previous generation, hence, it will be difficult for them to relocate to rural areas in the future.

In 2000s, many Chinese scholars embarked on the investigation of migrant workers in order to better classify the new generation. The criteria are chronicled in Luo Xia and Wang Chunguang's The Causation and Action - Choice of the New Generation of Rural Floating Population (2003)", Zhao Fang's “The New Generation"- A Concept Which is Difficult to be Defined (2003) ${ }^{5}$, Wang Xingzhou's On the Group Features of the New Generation of Rural Workers $(2008)^{6}$ and Fu Ping's The Inverted U Shaped Trajectory and the Social Mobility of New Generation Peasant Workers (2009) ${ }^{7}$. These discussions about defining migrant workers fall into two categories; one is a comprehensive study focusing on the classification and the group characteristics of the whole migrant worker community; another studies the characterization of the social group, emphasizing specific aspects of the new generation of migrant workers, such as their mobility and employment, life and consumption, marriages and crimes, social recognition and urban integration, and social welfare and political aspirations.

\footnotetext{
${ }^{4}$ Xia Luo and Chunguang Wang, "The Causation and Action - Choice of the New Generation of Rural Floating Population," Zhejiang Social Sciences 19, no. 1 (2003): 111-115.

${ }^{5}$ Fang Zhao, "'The New Generation'- A Concept Which is Difficult to Be Defined," Sociological Studies 18, no. 6 (2003): 71-83.

${ }^{6}$ Xingzhou Wang, "On the Group Features of the New Generation of Rural Workers - Taking the Pearl Delta for Example," Journal of Guangxi University for Nationalities 30, no. 4 (2008): 51-56.

7 Ping Fu and Youcai Tang, "The Inverted U Shaped Trajectory and the Social Mobility of New Generation Peasant Workers," Zhejiang Social Sciences 25, no. 12 (2009): 41-47.
} 
In regards to the comprehensiveness of the social group, Yang Juhua (professor at Department of Population and Development Research Center, Renmin University of China) summarizes seven major features of the floating population: low incomes, low occupational prestige, low social status, long working hours, insufficient welfare benefits, and poor accommodations. ${ }^{8}$ Yang explains that the more the migrant workers desire to fit into cities, the greater the conflict between the reality and the ideal. Therefore, rural workers are always trapped in a psychological cycle of desire, despair, and retreat. According to Wang Xingzhou (2008), the third generation of migrant workers tend to accumulate more human capital $^{9}$ and a better individual quality of life; He describes them as an ambitious and aspiring group who crave fair market discipline, liberty, equality and independence. ${ }^{10}$ Chinese economists Wu Hongyu and Xie Guoqiang (2006) point out that with better educational attainment, the new generation of peasant workers push themselves to integrate faster into urban life. They constantly change careers in a short period of time, aiming for higher incomes and a better quality of life. ${ }^{11}$

\footnotetext{
8 Juhua Yang, "The Misunderstanding of the Floating Population," Population Research 34, no. 2 (2010): 44-53.

${ }^{9}$ Human capital is defined in the Oxford English Dictionaries as "the skills, knowledge, and experience possessed by an individual or population, viewed in terms of their value or cost to an organization or country." "Human Capital - Definition of Human Capital in Oxford English Dictionaries," Oxford English Dictionaries, accessed August 01, 2017, https://en.oxforddictionaries.com/definition/human_capital.

10 Xingzhou Wang, "On the Group Features of the New Generation of Rural Workers - Taking the Pearl Delta for Example," Journal of Guangxi University for Nationalities 30, no. 4 (2008): 51-56.

${ }^{11}$ Hongyu Wu and Guoqiang Xie, "The Characteristics, Interest Claims and Role-Changing of Workers of the New Generation from the Rural: A Survey in Tangxia Town, Dongguan," South China Population 21, no. 2 (April 3, 2006): 21-31.
} 
Although this special social group is rooted in China, it also attracts western scholars' attention. Leslie T. Chang, a former correspondent for the Wall Street Journal, provides a new insight into the female migrant workers of the third generation. For the first two decades (1980-2000), male peasant workers had dominated the field, while the third generation is more gender balanced with a considerable number of female workers actively involved in various industries. In Factory Girls: From Village to City in a Changing China (2009), Chang sheds light on the advent and transition of Chinese female workers during China's social reform. With two particular case studies, the author demonstrates the hardship and struggle of female migrant workers. After three years' observation, Chang discovered a typical career pattern of these female workers. Almost every female manager Chang interviewed started her career as a production worker. Mandated by factory owners, they worked countless hours in hazardous conditions in order to increase profits for the company. Additionally, for these urban migrants, lying about education and work experience become a requisite for getting ahead. ${ }^{12}$

In terms of the characterization of the new generation who were born in early 1990s, Luo Xia and Wang Chunguang (2003) assert that different from their parents, rural workers nowadays have higher and higher expectations for their future when their goals are no longer limited to the needs of survival. ${ }^{13}$ In the

\footnotetext{
12 Leslie T Chang, Factory Girls: From Village to City in a Changing China (New York, NY: Spiegel \& Grau, 2009).

${ }^{13}$ Xia Luo and Chunguang Wang, "The Causation and Action - Choice of the New Generation of Rural Floating Population," Zhejiang Social Sciences 19, no. 1 (2003): 111-115.
} 
view of Pan Jiqing (2009), editor of Modern Youth Studies, the new generation of peasant workers consider rural-urban migration as a life-changing choice. In other words, they are willing to sacrifice everything to achieve success. However, due to deficiencies in the urban-rural dual system and their lack of knowledge and skills, migrant workers have difficulty in protecting their individual rights and interests. ${ }^{14}$

The social identity of migrant workers is another broadly discussed issue. Hu Xiaohong (2008) reveals that the third generation of migrant workers tend to be ambiguous, uncertain and self-contradictory when it comes to the recognition and evaluation of self-identity in the society. Hu adds that these inner dilemmas and anxieties are attributed to the restless minds of the third generation. ${ }^{15}$ They identify themselves as a particular social group yet can refer to neither the urban nor rural communities to which they belong. ${ }^{16}$ According to Chinese economists Liu Chuanjiang and Wang Guohua (2009), this social group is also denoted as "the marginals" and "the amphibians" of cities where they can neither belong nor retreat. $^{17}$

\footnotetext{
${ }^{14}$ Jiqing Pan, Haiyan Tan, and $\mathrm{Na} \mathrm{Li}$, "Rural and Urban Shift of the New Generation of Migrant Workers with an Analysis of Their Employment Experience," Contemporary Youth Research 27, no. 2 (2009): 42-48.

${ }^{15}$ Xiaohong Hu, "New Generation Migrant Laborers' Dilemma in Self Identity in Social Memory A Case Study on Many New Generation Migrant Laborers in S County," China Youth Study 20, no. 9 (September 5, 2008): 42-46.

${ }^{16}$ Chuanjiang Liu, "China's New Generation of Migrant Workers: Characteristics, Problems and Counter Measures," Population Research 34, no. 2 (2010): 34-56.

17 Guohua Wang, "Logical Comparison and Empirical Study on Cultural Adaption of Migrant Workers in Two Generations," Northwest Population Journal 30, no. 5 (2009): 47-50.
} 
In the field of Chinese documentary photography, migrant workers have been gradually taken as a significant subject, revealing the little-known details of the life of Chinese rural laborers. Hou Dengke's Mai Ke (2000) ${ }^{18}$, Zeng Huang and He Beili's Portraits of Chinese Peasants': Story of 900 Million People (2004) ${ }^{19}$, Chen Xiaobo's Hou Dengke - Flew Migratory Birds (2007) ${ }^{20}$ and Tang Haowu's Peasant Workers (2011) ${ }^{21}$, in the form of photographic monographs, uncover different kinds of complex social problems and cultural conflicts this marginalized social group are exposed to during China's rapid spread of urbanization. These photographers, with personalized perspectives as insiders, reveal the reality and spiritual needs of the new generation of migrant workers.

Illustrated press reports, in this case, are considered as the most effective way to raise social awareness and concern for migrant workers. Focusing on the Events with Lens, Provoking Thoughts with Pictures - How to Describe Migrant Workers' Retreat with Photography (2009) ${ }^{22}$, and Outsiders' Struggle for Survival - Image of Migrant Workers in News Coverage in Shanghai (2009) ${ }^{23}$, offer an in-depth analysis of the photographs, from different Chinese news agencies, which documented the stories of migrant workers from different entry points and

\footnotetext{
${ }^{18}$ Hou Dengke, the benchmark-setting photographer in Chinese contemporary documentary photography, spent 11 years (1989-2000) in northern Shaanxi, China documenting the life of "Mai Ke," peasant workers employed to help reap wheat.

${ }^{19}$ Beili He and Huang Zeng, Portraits of Chinese Peasants': Story of 900 Million People (Beijing: China Social Science Press, 2004).

${ }^{20}$ Xiaobo Chen, Hou Dengke - Flew Migratory Birds (Beijing: China Renmin University Press, 2007).

${ }^{21}$ Haowu Tang, Peasant Workers (Shanghai: Shanghai Jinxiu Article Press, 2011).

${ }^{22}$ Fawei Yang and Jinyi Yu, "Focusing on the Events with Lens, Provoking Thoughts with Pictures - How to Describe Migrant Workers' Retreat with Photography," Press Outpost 16, no. 4 (2009): 34-35.

23 Tingting Wu and Shilin Zhao, "Outsiders' Struggle for Survival - Image of Migrant Workers in News Coverage in Shanghai," Journalism Lovers 24, no. 2 (2009): 10-12.
} 
perspectives. ${ }^{24}$ These articles also demonstrate how news photography embodies the social value of migrant workers.

Comprised of over three hundred photographs, Ouyang Xingkai's exhibition catalogue People's Road (2012) provides a visual interpretation of the living space of migrant workers. On Renmin Road, Changsha, Hunan Province, hundreds of peasant workers squeeze into a three-story building divided into 190 rooms in a total floor area of only 1680 square meters. In other words, the available space for each tenant is merely four square meters. Photographs from People's Road depict the relationship between the people at the bottom of the society and the urban space in the development of urbanization. Hao Meijin (2014) comments that for these migrant workers, the crowed narrow visual space in People's Road implies the bondage and oppression of urbanization. Essentially, the power relations between human and space contributes to the construction of migrant workers' marginalized social status. ${ }^{25} \mathrm{He}$ Guangyi's Sufferings and Passions, Love and Hate - Documentary Photographer Hou Dengke and Study on His Works (2009) ${ }^{26}$ and Li Yunlei's Migrant Workers: A Fulcrum of The World - Tang Haowu's Migrant Workers Collection (2008) ${ }^{27}$, in the form of critical essays, provide an in-depth analysis and interpretation of the representative photographers (Hou Dengke and Tang Haowu) specializing in

\footnotetext{
${ }^{24}$ Photographs of migrant workers were used to illustrate the stories in the new coverage.

25 Meijin Hao, "Analysis of Migrant Workers' Documentary Photography from the Perspective of Visual Culture," Radio and TV Journal 7, no. 5 (2014): 75-77.

${ }^{26}$ Guangyi He, "Sufferings and Passions, Love and Hate - Documentary Photographer Hou Dengke and Study on His Works" (master's thesis, Northwest University, 2009).

${ }^{27}$ Yunlei Li, "Migrant Workers: A Fulcrum of The World. Tang Haowu's Migrant Workers

Collection," Photographers of China 15, no. 2 (2008): 80-85.
} 
documenting migrant workers. This literature includes both individual case studies and general studies, and discusses the aesthetic value and the realistic significance of the photographs of migrant workers.

Photographic albums of migrant workers constitute a major part of industrial and cultural heritage in China. The preservation and care of photographic albums have become increasingly prominent in museums, galleries, private archives and photographic institutions. Published on The Library of Congress website in 1991, "Preservation of Scrapbooks and Albums" addresses various preservation basics, including the reasonable range of temperature and relative humidity control for the useful life span of albums, the influence of excessive exposure of albums to light, the physical storage requirements of albums of different sizes, their handling, conservation treatments and reformatting. ${ }^{28}$ Similarly, "Preserving and Protecting Photographs" (2011) from The American Museum of Photography analyzes different kind of unsafe materials in albums and provides recommendations concerning the storage of albums accordingly. ${ }^{29}$ In terms of housing methods, Carlson and Brown's "Boxes for the Protection of Books: Their Design and Construction" (1994) ${ }^{30}$ and Denise Psaila's "Design and Materials for

\footnotetext{
${ }^{28}$ Barbara Zucker, "Preservation of Scrapbooks and Albums," Preservation Basics: A National Cooperative Information Project, Leaflet no. 1 (Washington, D.C.: Library of Congress, 1991), accessed June 14, 2017, http://www.loc.gov/preservation/care/scrapbk.html.

29 "Preserving and Protecting Photographs: A Buyer's Guide," The American Museum of Photography, 2011, accessed June 14, 2017.

http://www.photographymuseum.com/archival.html\#index.

${ }^{30}$ Lage Carlson and Margaret R. Brown, Boxes for the Protection of Books: Their Design and Construction (Washington, D.C.: Library of Congress, 1994).
} 
Photographic Housing" (2006) ${ }^{31}$ are the most resourceful guides, which introduce and compare various housing strategies for different photographic materials with detailed instructions, illustrations and diagrams. This literature was extremely helpful to me in devising the best preservation strategy for this migrant worker album.

Because of the complexity and uniqueness of photographic albums, all treatments are usually carried out on a case-by-case basis. Conservation of Scrapbooks and Albums (2000) ${ }^{32}$ introduces nine case studies relating to photographic albums conducted by different institutions, such as the Arizona State Museum (ASM), the Harry Ranson Humanities Research Center (HRHRC), the Art Institute of Chicago, and the Library of Congress. In the form of academic essays and interim reports, this volume investigates the history of the imagery in albums, describes the structure, materiality and condition of albums, and analyzes the problem and solutions encountered in the treatment of albums.

In conclusion, migrant workers have gradually become a significant subject matter in the field of Chinese literature research and photographic research. However, the archival and published sources on photography about Chinese migrant workers are still limited and studies on photography about migrant

\footnotetext{
${ }^{31}$ Denise Psaila, "Design and Materials for Photographic Housing" (master's thesis, Ryerson University, 2006).

32 Shannon Zachary, Conservation of Scrapbooks and Albums: Postprints of the Book and Paper Group/Photographic Materials Group Joint Session at the 27th Annual Meeting of the American Institute for Conservation of Historic and Artistic Works, June 11, 1999, St. Louis, Missouri (Washington, D.C.: Book and Paper Group [and] Photographic Materials Group, American Institute for Conservation of Historic and Artistic Works, 2000).
} 
workers are relatively fragmented and lack a panoramic and systematic research methodology. Lastly, the literature on preservation and conservation of photographic albums provided extreme useful information for the development and application of this applied thesis. 


\section{Chapter 2. Overview of the Album}

Made in December 2015, the Chinese photographic album that is the object of this thesis is entitled Work. The Manufacturing Industry in Dongguan: Real-life Stories of Migrant Workers, ${ }^{33}$ and signed on the front cover by the photographer Zhan Youbing. The album's handwritten title page includes the title, the date, and the photographer's signature, and is then followed by a two-page introduction Zhan wrote in pencil in simplified Chinese ${ }^{34}$. The album comprises fifty fiberbased D2T2 prints ${ }^{35}$, each approximately $34 \times 23 \mathrm{~cm}$ (13.39 x 9.05 inches), and other objects relating to Chinese migrant workers including a discarded photograph found by Zhan, an electronic component used for the production of hard disk heads, a clock card, a notice, a page of a handwritten canteen menu, a page of handwritten graffiti and a page of corporate seals.

The overall size of the album is $42.5 \times 30 \times 6 \mathrm{~cm}$ ( $16.73 \times 11.81 \times 2.36$ inches), which is by far the largest within Zhan's photographic collection at the AMC. Both front and back covers are made out of kraft paper covered with denim. The combination of these materials, in my interpretation, is a representation of the

\footnotetext{
${ }^{33}$ Translated by the author from Chinese simplified character: “打工” (Work) (reads as: Da Gong); “东莞制造业” (The Manufacturing Industry in Dongguan) (reads as: Dongguan Zhizao Ye); “农民 工生活实录” (Real-Life Stories of Migrant Workers) (reads as: Nong Min Gong Shenghuo Shilu).

34 The translated introduction can be found in Chapter 3.1

${ }^{35}$ D2T2 is the abbreviation of Dye Diffusion Thermal Transfer. "Heat applied pixel by pixel from a thermal array to a donor ribbon causes dyes to diffuse into the receptor coating of the media." Martin Jürgens, The Digital Print: Identification and Preservation (Los Angeles: Getty Conservation Institute, 2009), 144.
} 
manufacturing industry or the product of Chinese migrant workers' labour. ${ }^{36}$ The title "Work" in Chinese characters is custom-made with black cloth and stitched on the front cover. In terms of structure, the album is bound with white cotton strings on the left hand side and opens from right to left, as in the Western style. Each page of the album contains one photograph or one object mounted on kraft paper, with captions written horizontally in Chinese by Zhan, primarily introducing the subject of each image. Interestingly, the binding of this album is similar to the traditional Chinese stitched bookbinding, which first appeared in the Ming Dynasty (the sixteen century). ${ }^{37}$ Originally, Chinese stitched books were bound at the right and opened from left to right. This structure was decided by the layout of the contents which, in ancient China, were written vertically. However, this photographic album was made in 2015 and adopted the modern writing style. Therefore, the layout of the texts is no longer vertical but horizontal, which also explains why its bookbinding is on the opposite side, which is different from the traditional one.

\subsection{Provenance}

In October 2016, purchasing agent Ruben Lundgren purchased this album for AMC's main archive in Holland Park, London, as part of a set of 30 handmade

\footnotetext{
${ }^{36}$ With more than fifty thousand textile factories, China has become the world's largest textile manufacturer and exporter. Denim is one of the most exported textile product of China representing the rapid development of China's economy and the massive labour force behind the textile industry. Cheng Xu, "China Produces More Than Half of the World's Textile," Xinhua News, February 09, 2016, accessed August 02, 2017, http://news.xinhuanet.com/fortune/201602/09/c_1118017462.htm.

${ }^{37}$ Hu Yang, Chinese Publishing (Cambridge: Cambridge University Press, 2012), 76.
} 
photographic albums by Zhan Youbing. Lundgren is a Dutch photographer who lives and works in London and Beijing, respectively. The history of the AMC acquisition goes back to November $21^{\text {st }}, 2015$, when Zhan, as the Vice President of the Changan Photography Association, was invited to the Eleventh Lianzhou International Photography Exhibition in Lianzhou, Guangdong. On this occasion, Martin Parr ${ }^{38}$ and WassinkLundgren ${ }^{39}$ co-curated a book fair called "Chinese Photography Fair" in order to present modern China via the lens of Chinese photographers. Zhan brought eight of his handmade photographic albums to the exhibition and showed them to Ruben Lundgren. Lundgren was attracted to these albums at first glance and later recommended them to Martin Parr. Three minutes after reviewing the albums, Parr made the first purchase of one of Zhan's handmade albums Work Break. ${ }^{40}$ Since then, more and more private collectors and archives have purchased Zhan's work, and the AMC is one of them buying a collection of 30 albums. Later in January 2017, the collection of 30 handmade photographic albums by Zhan was shipped to the AMC in Toronto for cataloguing and translation.

\footnotetext{
${ }^{38}$ Martin Parr is a well-known British Documentary Photographer who focuses on documenting the social classes of England. "Parr is an exemplary photographic figure at the beginning of the twenty-first century. He is a founder of the New European Colour Photography School, yet is also a member of Magnum, photojournalism's premier agency." Gerry Badger, "Ruthless Courtesies: The Making of Martin Parr," in The Pleasures of Good Photographs: Essays (New York: The Aperture Foundation, 2010), accessed August 02, 2017, http://www.gerrybadger.com/wpcontent/uploads/2012/03/ParrByBadger.pdf.

39 WassinkLundgren is a company of photographic books, co-founded by Dutcher photographers Thijs groot Wassink and Ruben Lundgren. WassinkLundgren. "About," WassinkLundgren, accessed June 21, 2017, http://www.wassinklundgren.com/about/.

${ }^{40}$ Shouxin Tang, "Changan: Zhan Youbing's Handmade Album was Collected by Martin Parr," Southcn.com, November 24, 2015, accessed April 10, 2017, http://dg.southcn.com/content/201511/24/content_137588899.htm.
} 


\subsection{Exhibition History}

Before the AMC's acquisition, Zhan's migrant worker collection had been exhibited twice in Mainland China. In May 2016, it was first shown in "the $12^{\text {th }}$ China International Cultural Industries Fair" in Shenzhen. As stated in the preface of the exhibition catalogue, Zhan's photographs showed the real faces and state of migrant workers. ${ }^{41}$ Later in November, the collection was exhibited in the "2016 Taizhou Photography Biennial: ‘Encounter: Handmade Photo Albums.”"42 As photography critic Gu Zheng put it, Zhan comprehensively documented the lives of employees of the multinational foundry business, and gave a persuasive visual interpretation of the indifference of the production machines and the staff management in the factory setting. ${ }^{43}$

In late 2016, Zhan first introduced his migrant worker collection to the West. From November $1^{\text {st }}$ to December $9^{\text {th }}$ at the Weeks Gallery in Jamestown, New York, Zhan with American photographers Monika Garami, and Brian Ulrich, coexhibited a show called Made in China exploring the global supply chain of Chinese-made consumer goods.

\footnotetext{
${ }^{41}$ Shuiping Zhao, "The 12th China (Shenzhen) International Cultural Industries Fair," News.timedg.com, May 14, 2016, accessed April 09, 2017, http://news.timedg.com/201605/14/20419747.shtml.

42 Jinwen Shi, "A Glimpse at the 2016 Taizhou Photography Biennial," Meipian.cn, November 30, 2016, accessed April 09, 2017, https://www.meipian.cn/9mwery9.

${ }^{43}$ Zheng Gu, "Gu Zheng: How Does Photography Bear the Responsibility of the 'Post-Emmanuel Era'," Art.china.cn, September 17, 2014, accessed April 09, 2017, http://art.china.cn/voice/201409/17/content_7238351.htm.
} 
The director and curator of the gallery, Patricia Briggs stated, "Zhan's photographs reveal the faces of workers who are thousands of miles away from us but who, in a sense, touch our lives every day." ${ }^{44}$

44 "'Made in China' Opens Tuesday at JCC," The Post-Journal, October 28, 2016, accessed April 01, 2017, http://content.post-journal.com/?p=702419\%2F-Made-In-China--Opens-Tuesday-AtJCC.html. 


\section{Chapter 3. Catalogue of the Album}

\subsection{Translated introduction of the album}

\section{Documenting the Life and Work of Fellow Workers}

In late 1995, I was discharged from the People's Armed Police Corps of Sichuan and went back to my hometown Hubei. A week later, I set foot on a train to the South and began my journey as a migrant worker. After doing 102 push-ups in a row for the boss, I became the security guard of Shenzhen Lianwu Hotel. Three months later, I moved to Shenzhen Airport Hotel, still as a security guard, and my salary was raised from 450 yuan to 800 yuan. A year later, I became the Dean of General Affairs of Shenzhen Xixiang Baifa No.2 Toy Factory, in charge of personnel administration of the whole factory. Later on, I worked for hardware and electroplating factories and electronics factories for three years. After that, I quit several jobs and had been fired several times.

Constantly changing jobs, I was completely rootless and adrift, going with the flow of unemployment, from seeking a job to keeping a job. My mind was always occupied with survival.

In 2000, I was employed as a security supervisor at Dongguan Shili Technology and Electronics Factory, and spent 13 years in this large-scaled Hong Kong- 
funded enterprise, located in Dongguan, Guangdong. At first, I photographed for the factory newspaper with passion. However, my passion for photography had got out of hand to the extent that photography occupied all of my leisure time. I began by photographing various kinds of activities held at the factory, staff ID cards, and meetings. Gradually, I got bored. Then I tried photographing the daily life of my colleagues, and posted these photographs online. Surprisingly, the photographs attracted attention and comments from many photography teachers. I was encouraged and gained more confidence. Then I embarked on planning my shooting schedule. As I read (about photography), I started to think about my approach to documenting the life and work of colleagues and to document the development and changes in the industrial zone.

Since 2006, I have broken three cameras and have taken over three hundred thousand photographs of the life of migrant workers. In addition, I have collected a large quantity of recordings, videos and objects relevant to migrant workers.

I am a farmer, but I know nothing about farming. Having worked in the industrial zone for years, I am familiar with it and the daily life of fellow workers. The industrial zone is filled with adjacent factories processing imported supplied material. Owners of these enterprises are foreign businesspersons, while employees are all migrant workers. Always busy manufacturing on the assembly lines and working three hours overtime everyday, the youthful spirit of migrant workers has gone, just like the export of products. 
China's manufacturing industry is known around the world, while the migrant workers behind it are obscure.

We neglected the sunk $\operatorname{cost}^{45}$ by regarding the environmental resources, air, water and labor as inexhaustible. Yet we were constantly suffering from the retaliation of nature. We began to think of what lessons needed to be learnt in the developing process of urbanization. In this, photography has provided us with a clear mirror.

Migrant workers are peasants who are manufacturing industrial goods sold around the world. With cameras, I captured the life and work of myself and my fellow workers. I documented not only the manufacturing process of these industrial goods but also the reality of migrant workers, who manufacture industrial goods and are being treated as the means of production. The increase in capital improved the lifestyle of migrant workers, yet the role of these migrant workers as profit makers has never changed. What migrant workers sacrificed was not merely their labour; it was also their youth, health, familial love and even life. Eventually, they were relentlessly discarded as waste by the logic of capitalism.

\footnotetext{
45 "Sunk costs are costs which, once committed, cannot be recovered. Sunk costs arise because some activities require specialized assets that cannot readily be diverted to other uses," R. S. Khemani and D. M. Shapiro, "Glossary of Industrial Organisation Economics and Competition Law," Organization for Economic Co-operation and Development, 1993, accessed April 10, 2017, http://www.oecd.org/regreform/sectors/2376087.pdf.
} 
The photographs I have taken gradually take on the face of history. Let us face the past and reflect on the development of the future.

All photographs were taken in Changan Town, Dongguan, Guangdong Province. (Expect for those with specific location marks)

(Signed) Zhan Youbing Changan Town, Dongguan, December 2015 


\subsection{Introduction to the Catalogue}

The main purpose for this catalogue is to enhance the management of Zhan's collection at the AMC and to make this album more accessible to future researchers by translating the captions and the text from Chinese to English, and cataloguing the entire album according to AMC's cataloguing standards using the Art Systems software. In total, the catalogue comprises sixty records devised with sixteen fields, in which relevant information about each object is documented. A brief definition for each field is given below:

\section{Collection}

This field records the name of the collection that the album is assigned to.

\section{Index Number}

This field records the unique and permanent number assigned to each object in the collection. Each page of the album is catalogued as a single object. Since these objects are related to one another, their individual index numbers are sequential.

AMC's naming system: LFA 30427_30

a. LFA: The name of the collection: Lavis Fine Arts

b. 30427: The order of the item purchased in the collection 
c. "_n": Followed by the number of items within a group. For example, "_30" refers to the $30^{\text {th }}$ item in the group

d. "-": Stands for the views of the item,

E.g. LFA30427_30-v1: the back cover of the album, v=verso.

E.g. LFA30427_30-2: the inner cover of the album

\section{Title}

This field records the title of each object given by the photographer.

\section{Artist}

This field records the name of the creator of the object.

\section{Location}

This field records the storage location of the object. Abbreviation is often used in this field for convenience. For example, "65QSW21" refers to the address, 65 Queen Street West, 21 ${ }^{\text {st }}$ floor.

\section{Category}

This field records the division of objects with particular shared characteristics. For example, the category "albums" refers to bound or loose-leaf sets of pages with photographic prints.

\section{Medium}


This field is used to describe the physical materiality of the object or to identify the photographic process and reference techniques of the prints and the material of its support.

\section{Condition}

This field describes the current condition of the prints in the albums, as well as the condition of the supports. First, describe the general condition of the object: "Excellent," "Good," "Fair," or "Poor," followed by the specific kinds of deterioration of the print or the support: abrasion, accretion, adhesive residue, channeling, cockling, crackling, crease, dent, discoloration, embrittlement, scratch, tear, yellowing, wear.

\section{Date of Origin}

This field records the date of the creation of the object, which is not always given by the creator. If the exact date is unknown, use a circa date form, e.g. ca. December 2015

\section{Place of Origin}

This field records the place of the creation of the object.

\section{Description}

This field provides a description of the object, which is to help the viewer visualize the object by focusing on its most prominent features. This field also provides explanation of the title as additional reference. 


\section{Size}

This field records the physical dimensions (height and width) of the object measured in centimeters, followed by inches in parentheses.
a. Image
Measurement of the image only
b. Mount
Measurement of the support of the object

\section{Signature/Inscriptions}

This field records any printed or handwritten information (title, date, signature, stamp etc.) on the recto (front) and the verso (back) of the object or its support. (i.e. Titled and dated, in pencil, au mount recto)

\section{Purchased From}

This field records the provenance and the previous ownership of the object before its acquisition at the AMC.

\section{Date of Acquisition}

This field records the date of the object purchased by AMC.

\section{AMC Number}


This field records the number assigned to an intact object. The entire album is considered as one object, therefore each page of the album share the same AMC number. 


\subsection{Catalogue}

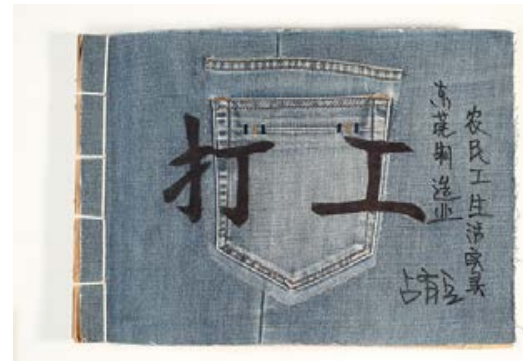

\section{Catalogue 1}

Collection: Lavis Fine Arts

Index No.: 30427_30

Title: Work. The Manufacturing Industry in Dongguan: Real-Life Stories of Migrant Workers.

Artist: Zhan Youbing

Location: 65QSW21 - 65 Queen Street West, 21 ${ }^{\text {st }}$ floor

Category: Albums

Medium: Denim photo album with embossed title contains 50 D2T2 prints and other objects related to Chinese migrant workers

Condition: Excellent

Date of Origin: December 2015

Place of Origin: Dongguan, Guangdong, China

Description: The album comprises 50 photographs of migrant workers taken by Zhan, two-pages of introduction written in Chinese in pencil, and objects relevant to migrant workers (including a discarded photograph found by Zhan, an electronic component, a clock card, a notice, a page of a handwritten canteen menu, a page of handwritten graffiti and a page of corporate seals)

Size: Images: $34.5 \times 23 \mathrm{~cm}(13.58 \times 9.05$ inches $)$

Mounts: $42 \times 29.6 \mathrm{~cm}(16.53 \times 11.65$ inches $)$

Signature/Inscriptions: Titled, dated and signed, in ink, au recto

Purchased From: WassinkLundgren (Ruben Lundgren)

Date of Acquisition: 20 October 2016

AMC No.: AMC 16525 


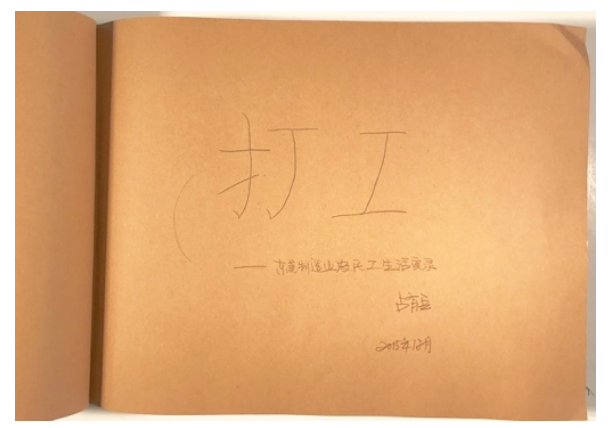

\section{Catalogue 2}

Collection: Lavis Fine Arts

Index No.: 30427_30-2

Title: Work. The Manufacturing Industry in Dongguan: Real-Life Stories of Migrant Workers.

Artist: Zhan Youbing

Location: 65QSW21 - 65 Queen Street West, 21 ${ }^{\text {st }}$ floor

Category: Albums

Medium: Kraft paper

Condition: Excellent, minor creases on top right corner

Date of Origin: December 2015

Place of Origin: Dongguan, Guangdong, China

Description: Title page

Size: 42 x $29.6 \mathrm{~cm}$ (16.53 x 11.65 inches)

Signature/Inscriptions: Titled, dated and signed, in pencil, au recto

Purchased From: WassinkLundgren (Ruben Lundgren)

Date of Acquisition: 20 October 2016

AMC No.: AMC 16525 


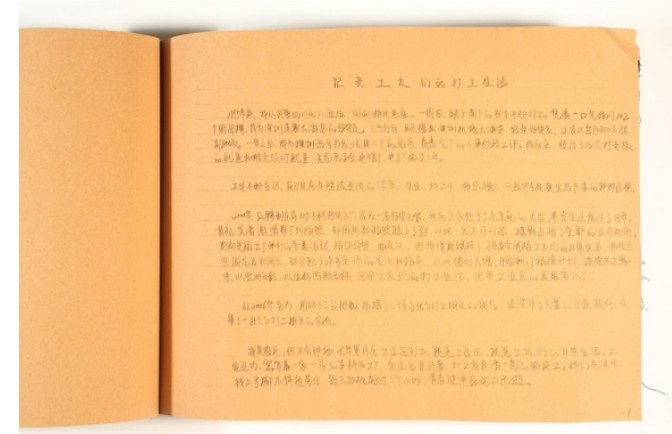

\section{Catalogue 3}

Collection: Lavis Fine Arts

Index No.: 30427_30-3

Title: Introduction (translated above page 20 - 23) entitled Documenting the Life and Work of Fellow Workers.

Artist: Zhan Youbing

Location: 65QSW21 - 65 Queen Street West, 21 ${ }^{\text {st }}$ floor

Category: Albums

Medium: Kraft paper

Condition: Excellent, minor creases on top right corner

Date of Origin: December 2015

Place of Origin: Dongguan, Guangdong, China

Description: Introduction written in Chinese in pencil by photographer

Size: 42 × $29.6 \mathrm{~cm}(16.53 \times 11.65$ inches $)$

Signature/Inscriptions: Titled and dated, in pencil, au recto

Purchased From: WassinkLundgren (Ruben Lundgren)

Date of Acquisition: 20 October 2016

AMC No.: AMC 16525 


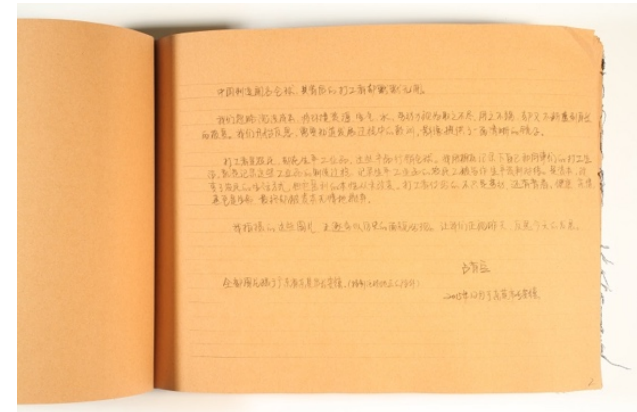

\section{Catalogue 4}

Collection: Lavis Fine Arts

Index No.: 30427_30-4

Title: Introduction (translated above page 20 - 23) entitled Documenting the Life and Work of Fellow Workers.

Artist: Zhan Youbing

Location: 65QSW21 - 65 Queen Street West, 21 ${ }^{\text {st }}$ floor

Category: Albums

Medium: Kraft paper

Condition: Excellent, minor creases on top right corner

Date of Origin: December 2015

Place of Origin: Dongguan, Guangdong, China

Description: Introduction written in Chinese in pencil by photographer

Size: $42 \times 29.6 \mathrm{~cm}$ (16.53 x 11.65 inches)

Signature/Inscriptions: Titled and dated and signed, in pencil, au recto

Purchased From: WassinkLundgren (Ruben Lundgren)

Date of Acquisition: 20 October 2016

AMC No.: AMC 16525 


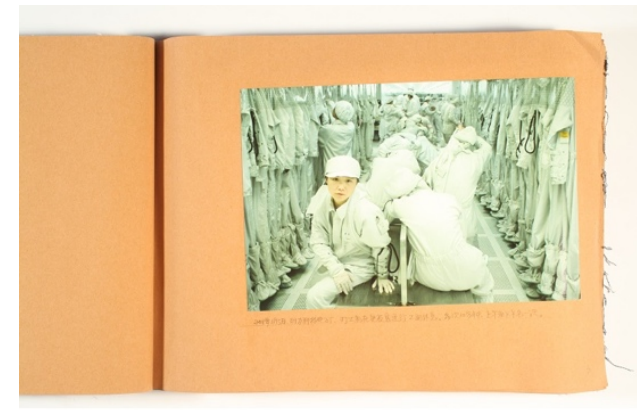

\section{Catalogue 5}

Collection: Lavis Fine Arts

Index No.: 30427_30-5

Title: Shili Technology and Electronics Factory. Migrant Workers Resting in the Changing Room: Two Ten-minute Breaks, One in the Morning and One in the Afternoon.

Artist: Zhan Youbing

Location: 65QSW21 - 65 Queen Street West, 21 ${ }^{\text {st }}$ floor

Category: Albums

Medium: D2T2 print mounted on kraft paper

Condition: Excellent, minor cockling

Date of Origin: 5 January 2011

Place of Origin: Dongguan, Guangdong, China

Description: None

Size: Image: $27.5 \times 18.3 \mathrm{~cm}(10.82 \times 7.20$ inches $)$

Mount: $42 \times 29.6 \mathrm{~cm}$ (16.53 x 11.65 inches)

Signature/Inscriptions: Titled and dated, in pencil, au mount recto

Purchased From: WassinkLundgren (Ruben Lundgren)

Date of Acquisition: 20 October 2016

AMC No.: AMC 16525 


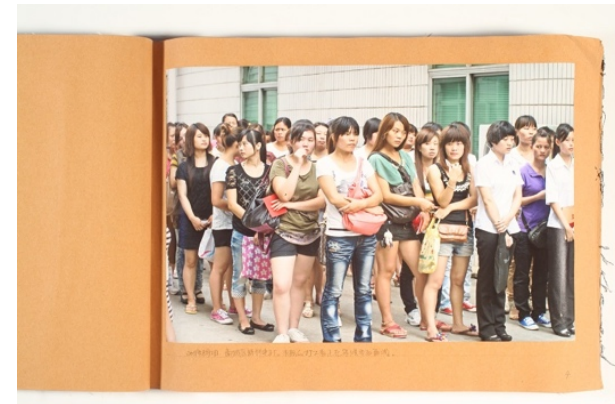

\section{Catalogue 6}

Collection: Lavis Fine Arts

Index No.: 30427_30-6

Title: Xinke Electronics Factory in Nancheng District. Job Seekers Waiting for Interviews.

Artist: Zhan Youbing

Location: 65QSW21 - 65 Queen Street West, 21 ${ }^{\text {st }}$ floor

Category: Albums

Medium: D2T2 print mounted on kraft paper

Condition: Excellent, minor cockling

Date of Origin: 2 August 2011

Place of Origin: Dongguan, Guangdong, China

Description: None

Size: Image: $34.5 \times 23 \mathrm{~cm}(13.58 \times 9.05$ inches $)$

Mount: $42 \times 29.6 \mathrm{~cm}(16.53 \times 11.65$ inches $)$

Signature/Inscriptions: Titled and dated, in pencil, au mount recto

Purchased From: WassinkLundgren (Ruben Lundgren)

Date of Acquisition: 20 October 2016

AMC No.: AMC 16525 


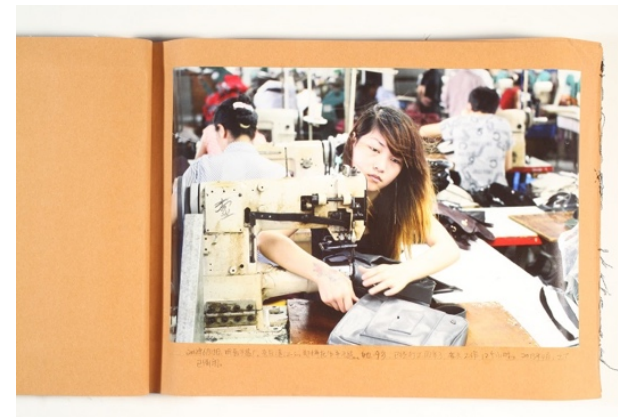

\section{Catalogue 7}

Collection: Lavis Fine Arts

Index No.: 30427_30-7

Title: Xinjin Handbag Factory. Zheng Ting, Who Comes from Zhanjiang, Manufacturing Handbags. She Is Nineteen Years Old and Has Been Working for Four Years, Twelve Hours Every Day. Factory Was Closed down in April 2013.

Artist: Zhan Youbing

Location: 65QSW21 - 65 Queen Street West, 21 ${ }^{\text {st }}$ floor

Category: Albums

Medium: D2T2 print mounted on kraft paper

Condition: Excellent, minor cockling

Date of Origin: 18 June 2012

Place of Origin: Dongguan, Guangdong, China

Description: None

Size: Image: $34.5 \times 22.8 \mathrm{~cm}(13.58 \times 8.98$ inches $)$

Mount: $42 \times 29.6 \mathrm{~cm}(16.53 \times 11.65$ inches $)$

Signature/Inscriptions: Titled and dated, in pencil, au mount recto

Purchased From: WassinkLundgren (Ruben Lundgren)

Date of Acquisition: 20 October 2016

AMC No.: AMC 16525 


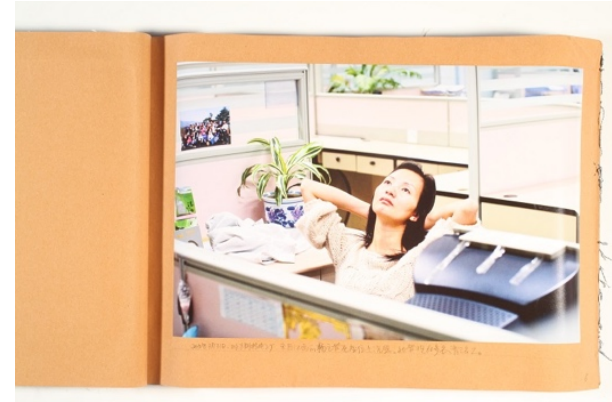

\section{Catalogue 8}

Collection: Lavis Fine Arts

Index No.: 30427_30-8

Title: Shili Technology and Electronics Factory. Executive Yang Lost in Her Thoughts While Sitting. She Manages Over Sixty Cleaners.

Artist: Zhan Youbing

Location: 65QSW21 - 65 Queen Street West, 21 ${ }^{\text {st }}$ floor

Category: Albums

Medium: D2T2 print mounted on kraft paper

Condition: Excellent, minor cockling

Date of Origin: 31 March 2010

Place of Origin: Dongguan, Guangdong, China

Description: None

Size: Image: $34.3 \times 23 \mathrm{~cm}(13.50 \times 9.05$ inches $)$

Mount: $42 \times 29.6 \mathrm{~cm}$ (16.53 x 11.65 inches)

Signature/Inscriptions: Titled and dated, in pencil, au mount recto

Purchased From: WassinkLundgren (Ruben Lundgren)

Date of Acquisition: 20 October 2016

AMC No.: AMC 16525 


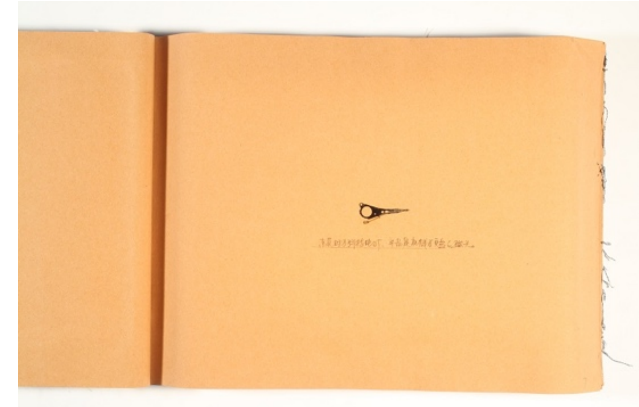

\section{Catalogue 9}

Collection: Lavis Fine Arts

Index No.: 30427_30-9

Title: Dongguan Shili Technology and Electronics Factory. A Magnetic Head of Mechanical Hard Drive.

Artist: Zhan Youbing

Location: 65QSW21 - 65 Queen Street West, 21 ${ }^{\text {st }}$ floor

Category: Albums

Medium: Electronic component mounted on kraft paper

Condition: Excellent

Date of Origin: Unknown

Place of Origin: Dongguan, Guangdong, China

Description: None

Size: Electronic component: $4.5 \times 1.8 \mathrm{~cm}$ (13.50 x 9.05 inches)

Mount: $42 \times 29.6 \mathrm{~cm}$ (16.53 x 11.65 inches)

Signature/Inscriptions: Titled in pencil, au mount recto

Purchased From: WassinkLundgren (Ruben Lundgren)

Date of Acquisition: 20 October 2016

AMC No.: AMC 16525 


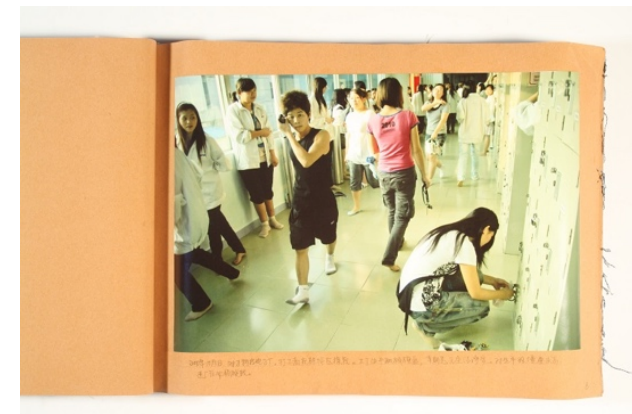

Catalogue 10

Collection: Lavis Fine Arts

Index No.: 30427_30-10

Title: Shili Technology and Electronics Factory. Migrant Workers Changing Shoes in the Shoe Locker Area. Factory Produces Mechanical Hard Drives. Manufacturing Shops, Which Are Dust-free Cleanrooms, Have High Standards for the Manufacturing Environment: It Is Mandatory to Take off Shoes after Entering the Factory.

Artist: Zhan Youbing

Location: 65QSW21 - 65 Queen Street West, 21 ${ }^{\text {st }}$ floor

Category: Albums

Medium: D2T2 print mounted on kraft paper

Condition: Excellent, minor cockling

Date of Origin: 1 October 2010

Place of Origin: Dongguan, Guangdong, China

Description: None

Size: Image: $34.5 \times 23 \mathrm{~cm}(13.58 \times 9.05$ inches $)$

Mount: $42 \times 29.6 \mathrm{~cm}$ (16.53 x 11.65 inches)

Signature/Inscriptions: Titled and dated, in pencil, au mount recto

Purchased From: WassinkLundgren (Ruben Lundgren)

Date of Acquisition: 20 October 2016

AMC No.: AMC 16525 


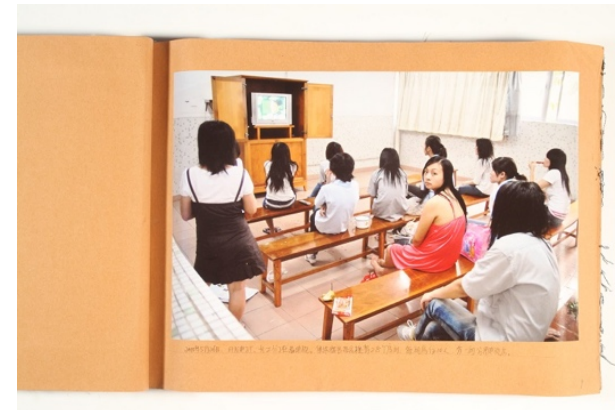

\section{Catalogue 11}

Collection: Lavis Fine Arts

Index No.: 30427_30-11

Title: Yuefa Electronics Factory. Female Workers Watching Television. There Are 28 Rooms on Each Floor of the Dormitory. Ten People Live in One Room: Only One Television Room.

Artist: Zhan Youbing

Location: 65QSW21 - 65 Queen Street West, 21 ${ }^{\text {st }}$ floor

Category: Albums

Medium: D2T2 print mounted on kraft paper

Condition: Excellent, minor cockling

Date of Origin: 26 May 2010

Place of Origin: Dongguan, Guangdong, China

Description: None

Size: Image: $34.5 \times 23 \mathrm{~cm}(13.58 \times 9.05$ inches $)$

Mount: 42 x $29.6 \mathrm{~cm}$ (16.53 x 11.65 inches)

Signature/Inscriptions: Titled and dated, in pencil, au mount recto

Purchased From: WassinkLundgren (Ruben Lundgren)

Date of Acquisition: 20 October 2016

AMC No.: AMC 16525 


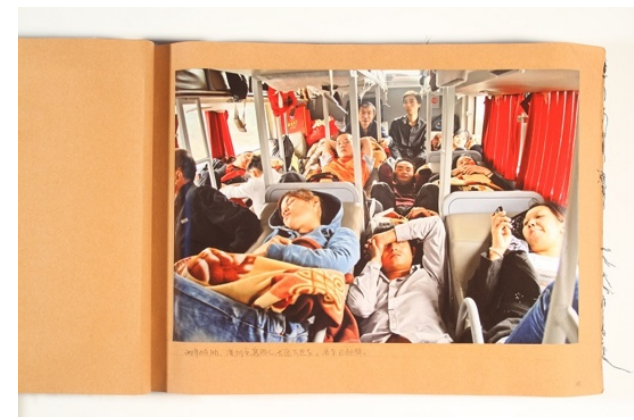

\section{Catalogue 12}

Collection: Lavis Fine Arts

Index No.: 30427_30-12

Title: Bound from Shenzhen to Xiangyang, the Long Distance Bus Is Already Overloaded.

Artist: Zhan Youbing

Location: 65QSW21 - 65 Queen Street West, 21 $1^{\text {st }}$ floor

Category: Albums

Medium: D2T2 print mounted on kraft paper

Condition: Excellent, minor cockling

Date of Origin: 30 October 2011

Place of Origin: Dongguan, Guangdong, China

Description: None

Size: Image: $34.3 \times 22.9 \mathrm{~cm}$ (13.50 x 9.01 inches)

Mount: $42 \times 29.6 \mathrm{~cm}(16.53 \times 11.65$ inches $)$

Signature/Inscriptions: Titled and dated, in pencil, au mount recto

Purchased From: WassinkLundgren (Ruben Lundgren)

Date of Acquisition: 20 October 2016

AMC No.: AMC 16525 


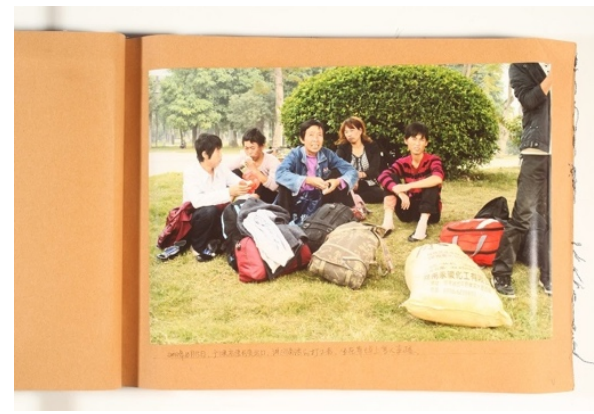

\section{Catalogue 13}

Collection: Lavis Fine Arts

Index No.: 30427_30-13

Title: Changan Exit of Guangshen Expressway. Migrant Workers, Who Speak Henanese, Sitting on the Lawn Waiting for Someone to Pick Them Up.

Artist: Zhan Youbing

Location: 65QSW21 - 65 Queen Street West, 21 ${ }^{\text {st }}$ floor

Category: Albums

Medium: D2T2 print mounted on kraft paper

Condition: Excellent, minor cockling

Date of Origin: 15 October 2012

Place of Origin: Dongguan, Guangdong, China

Description: None

Size: Image: 34.1 x $23 \mathrm{~cm}(13.42 \times 9.05$ inches $)$

Mount: 42 x $29.6 \mathrm{~cm}$ (16.53 x 11.65 inches)

Signature/Inscriptions: Titled and dated, in pencil, au mount recto

Purchased From: WassinkLundgren (Ruben Lundgren)

Date of Acquisition: 20 October 2016

AMC No.: AMC 16525 


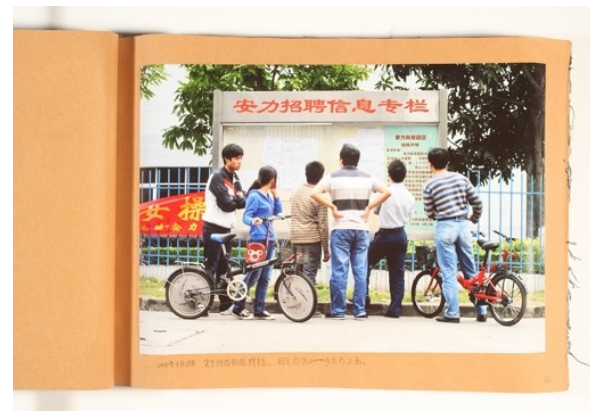

\section{Catalogue 14}

Collection: Lavis Fine Arts

Index No.: 30427_30-14

Title: Recruitment Bulletin Board in Anli Science and Technology Park. There are Over Twenty Thousand Migrant Workers in the Park.

Artist: Zhan Youbing

Location: 65QSW21 - 65 Queen Street West, 21 ${ }^{\text {st }}$ floor

Category: Albums

Medium: D2T2 print mounted on kraft paper

Condition: Excellent, minor cockling

Date of Origin: 18 April 2010

Place of Origin: Dongguan, Guangdong, China

Description: None

Size: Image: $34.3 \times 24.1 \mathrm{~cm}(13.50 \times 9.49$ inches $)$

Mount: $42 \times 29.6 \mathrm{~cm}$ (16.53 x 11.65 inches)

Signature/Inscriptions: Titled and dated, in pencil, au mount recto

Purchased From: WassinkLundgren (Ruben Lundgren)

Date of Acquisition: 20 October 2016

AMC No.: AMC 16525 


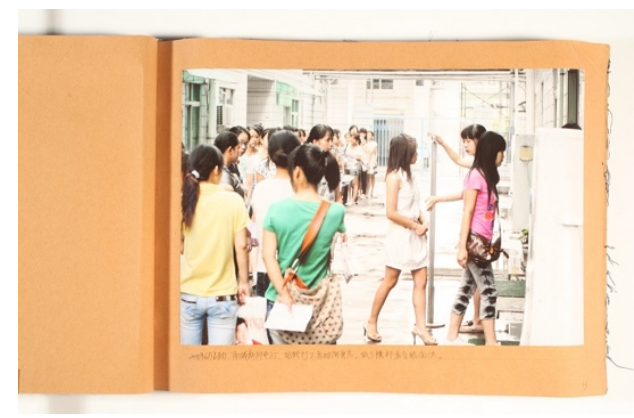

\section{Catalogue 15}

Collection: Lavis Fine Arts

Index No.: 30427_30-15

Title: Nancheng Xinke Electronics Factory. Job Seekers Measuring Height.

People Shorter Than the Cross Bar Will Be Eliminated.

Artist: Zhan Youbing

Location: 65QSW21 - 65 Queen Street West, 21 ${ }^{\text {st }}$ floor

Category: Albums

Medium: D2T2 print mounted on kraft paper

Condition: Excellent, minor cockling

Date of Origin: 28 June 2011

Place of Origin: Dongguan, Guangdong, China

Description: None

Size: Image: $34.2 \times 23 \mathrm{~cm}(13.46 \times 9.05$ inches $)$

Mount: $42 \times 29.6 \mathrm{~cm}$ (16.53 x 11.65 inches)

Signature/Inscriptions: Titled and dated, in pencil, au mount recto

Purchased From: WassinkLundgren (Ruben Lundgren)

Date of Acquisition: 20 October 2016

AMC No.: AMC 16525 


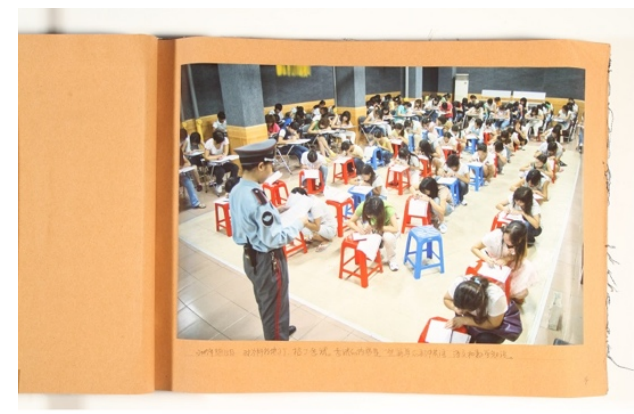

\section{Catalogue 16}

Collection: Lavis Fine Arts

Index No.: 30427_30-16

Title: Shili Technology and Electronics Factory. Placement Test: the Content of the Test Is Simple Knowledge of English, Chinese and Mathematics at the Junior High School Level.

Artist: Zhan Youbing

Location: 65QSW21 - 65 Queen Street West, 21 ${ }^{\text {st }}$ floor

Category: Albums

Medium: D2T2 print mounted on kraft paper

Condition: Excellent, minor cockling

Date of Origin: 15 August 2007

Place of Origin: Dongguan, Guangdong, China

Description: None

Size: Image: $34.4 \times 23 \mathrm{~cm}(13.54 \times 9.05$ inches $)$

Mount: $42 \times 29.6 \mathrm{~cm}$ (16.53 x 11.65 inches)

Signature/Inscriptions: Titled and dated, in pencil, au mount recto

Purchased From: WassinkLundgren (Ruben Lundgren)

Date of Acquisition: 20 October 2016

AMC No.: AMC 16525 


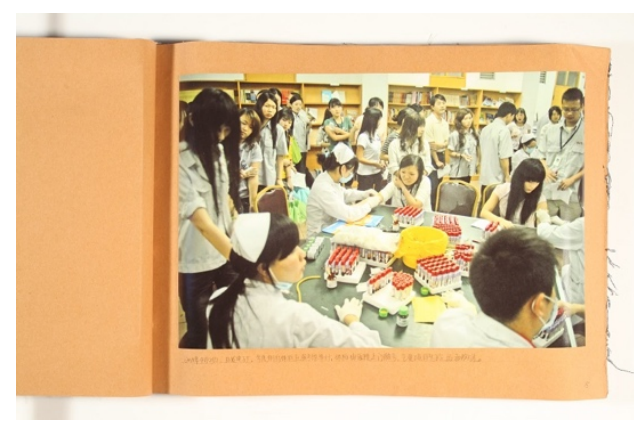

\section{Catalogue 17}

Collection: Lavis Fine Arts

Index No.: 30427_30-17

Title: Yuefa Electronics Factory. Annual Check-Up Held by Professional Medical Workers in the Library, Mainly Includes Blood Test and Chest X-Ray.

Artist: Zhan Youbing

Location: 65QSW21 - 65 Queen Street West, 21 ${ }^{\text {st }}$ floor

Category: Albums

Medium: D2T2 print mounted on kraft paper

Condition: Excellent, minor cockling

Date of Origin: 21 April 2010

Place of Origin: Dongguan, Guangdong, China

Description: None

Size: Image: $34.5 \times 23 \mathrm{~cm}(13.58 \times 9.05$ inches $)$

Mount: $42 \times 29.6 \mathrm{~cm}$ (16.53 x 11.65 inches)

Signature/Inscriptions: Titled and dated, in pencil, au mount recto

Purchased From: WassinkLundgren (Ruben Lundgren)

Date of Acquisition: 20 October 2016

AMC No.: AMC 16525 


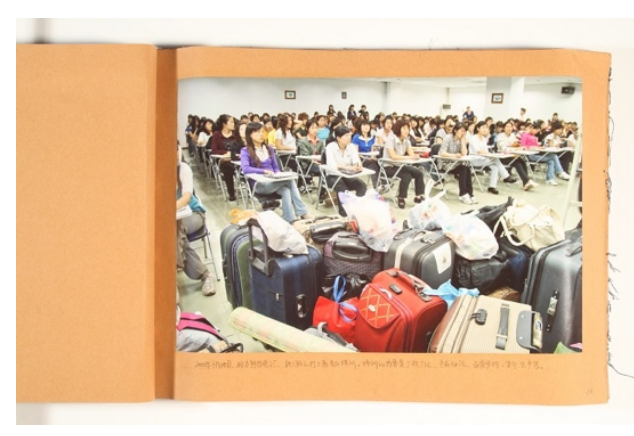

\section{Catalogue 18}

Collection: Lavis Fine Arts

Index No.: 30427_30-18

Title: Shili Technology and Electronics Factory. New Recruits Attending the Training Program on Factory Regulations and Disciplines, Product Information, Quality Management, and Production Safety.

Artist: Zhan Youbing

Location: 65QSW21 - 65 Queen Street West, 21 ${ }^{\text {st }}$ floor

Category: Albums

Medium: D2T2 print mounted on kraft paper

Condition: Excellent, minor cockling

Date of Origin: 20 March 2010

Place of Origin: Dongguan, Guangdong, China

Description: None

Size: Image: 34.4 x $23 \mathrm{~cm}(13.54 \times 9.05$ inches $)$

Mount: $42 \times 29.6 \mathrm{~cm}$ (16.53 x 11.65 inches)

Signature/Inscriptions: Titled and dated, in pencil, au mount recto

Purchased From: WassinkLundgren (Ruben Lundgren)

Date of Acquisition: 20 October 2016

AMC No.: AMC 16525 


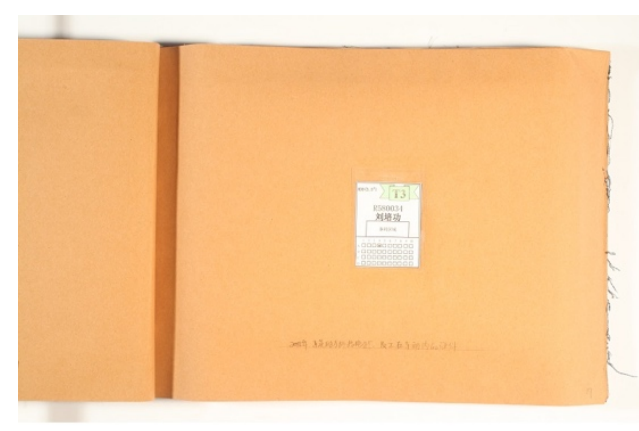

\section{Catalogue 19}

Collection: Lavis Fine Arts

Index No.: 30427_30-19

Title: Dongguan Shili Technology and Electronics Factory. Staff Card Used in Manufacturing Shops.

Artist: Zhan Youbing

Location: 65QSW21 - 65 Queen Street West, 21 ${ }^{\text {st }}$ floor

Category: Albums

Medium: Plastic card mounted on kraft paper

Condition: Excellent

Date of Origin: 2014

Place of Origin: Dongguan, Guangdong, China

Description: None

Size: Card: 6 × $8.4 \mathrm{~cm}(2.36 \times 3.30$ inches $)$

Mount: $42 \times 29.6 \mathrm{~cm}(16.53 \times 11.65$ inches $)$

Signature/Inscriptions: Titled and dated, in pencil, au mount recto

Purchased From: WassinkLundgren (Ruben Lundgren)

Date of Acquisition: 20 October 2016

AMC No.: AMC 16525 


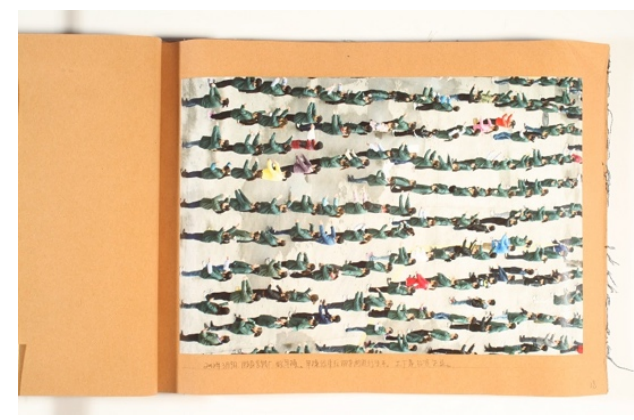

\section{Catalogue 20}

Collection: Lavis Fine Arts

Index No.: 30427_30-20

Title: Sheng Baiji Shoe Factory. Mandatory Morning Exercises. Staff Have to Go Back to Work in Manufacturing Shops After Morning Exercises. The Factory Is a Taiwan-Capital Enterprise.

Artist: Zhan Youbing

Location: 65QSW21 - 65 Queen Street West, 21 ${ }^{\text {st }}$ floor

Category: Albums

Medium: D2T2 print mounted on kraft paper

Condition: Excellent, minor cockling

Date of Origin: 9 March 2012

Place of Origin: Dongguan, Guangdong, China

Description: None

Size: Image: $34.3 \times 23 \mathrm{~cm}(13.50 \times 9.05$ inches $)$

Mount: $42 \times 29.6 \mathrm{~cm}$ (16.53 x 11.65 inches $)$

Signature/Inscriptions: Titled and dated, in pencil, au mount recto

Purchased From: WassinkLundgren (Ruben Lundgren)

Date of Acquisition: 20 October 2016

AMC No.: AMC 16525 


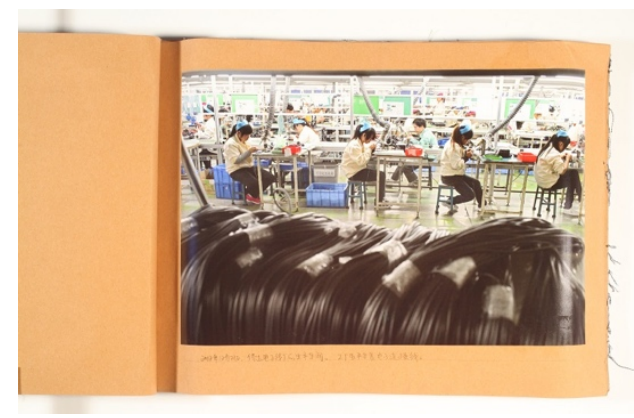

\section{Catalogue 21}

Collection: Lavis Fine Arts

Index No.: 30427_30-21

Title: Manufacturing Shop of Qiaoda Electronic Cable Factory. Factory Produces Different Kinds of Electronic Cables.

Artist: Zhan Youbing

Location: 65QSW21 - 65 Queen Street West, 21 $1^{\text {st }}$ floor

Category: Albums

Medium: D2T2 print mounted on kraft paper

Condition: Excellent, minor cockling

Date of Origin: 21 December 2012

Place of Origin: Dongguan, Guangdong, China

Description: None

Size: Image: 34.4 × $23 \mathrm{~cm}$ (13.54 x 9.05 inches)

Mount: $42 \times 29.6 \mathrm{~cm}(16.53 \times 11.65$ inches $)$

Signature/Inscriptions: Titled and dated, in pencil, au mount recto

Purchased From: WassinkLundgren (Ruben Lundgren)

Date of Acquisition: 20 October 2016

AMC No.: AMC 16525 


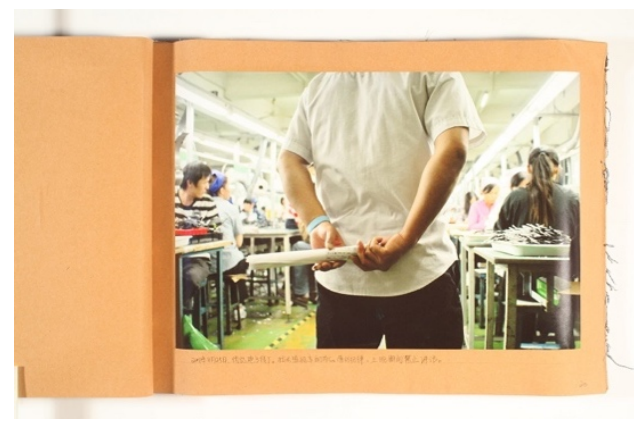

\section{Catalogue 22}

Collection: Lavis Fine Arts

Index No.: 30427_30-22

Title: Qiaoda Electronic Cable Factory. Lazhang Is Monitoring Labor Discipline in the Manufacturing Shop. Speaking Is Not Allowed during Work.

Artist: Zhan Youbing

Location: 65QSW21 - 65 Queen Street West, 21 ${ }^{\text {st }}$ floor

Category: Albums

Medium: D2T2 print mounted on kraft paper

Condition: Excellent, minor cockling

Date of Origin: 25 November 2015

Place of Origin: Dongguan, Guangdong, China

Description: None

Size: Image: $34.4 \times 23 \mathrm{~cm}(13.54 \times 9.05$ inches $)$

Mount: $42 \times 29.6 \mathrm{~cm}$ (16.53 x 11.65 inches)

Signature/Inscriptions: Titled and dated, in pencil, au mount recto

Purchased From: WassinkLundgren (Ruben Lundgren)

Date of Acquisition: 20 October 2016

AMC No.: AMC 16525 


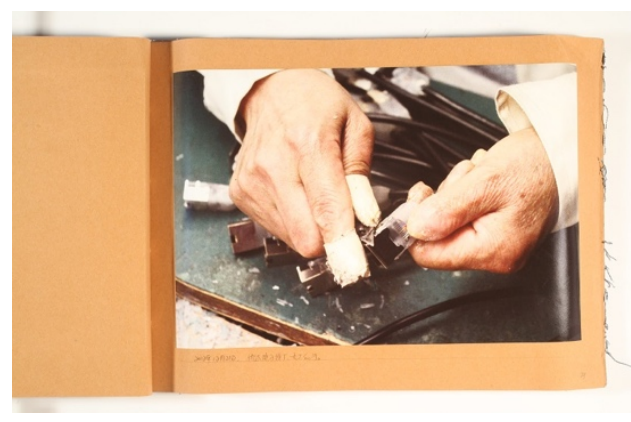

\section{Catalogue 23}

Collection: Lavis Fine Arts

Index No.: 30427_30-23

Title: Qiaoda Electronic Cable Factory. Hands of Female Worker.

Artist: Zhan Youbing

Location: 65QSW21 - 65 Queen Street West, 21 ${ }^{\text {st }}$ floor

Category: Albums

Medium: D2T2 print mounted on kraft paper

Condition: Excellent, minor cockling

Date of Origin: 21 December 2012

Place of Origin: Dongguan, Guangdong, China

Description: None

Size: Image: $34.5 \times 23.2 \mathrm{~cm}(13.58 \times 9.13$ inches $)$

Mount: 42 x $29.6 \mathrm{~cm}(16.53 \times 11.65$ inches $)$

Signature/Inscriptions: Titled and dated, in pencil, au mount recto

Purchased From: WassinkLundgren (Ruben Lundgren)

Date of Acquisition: 20 October 2016

AMC No.: AMC 16525 


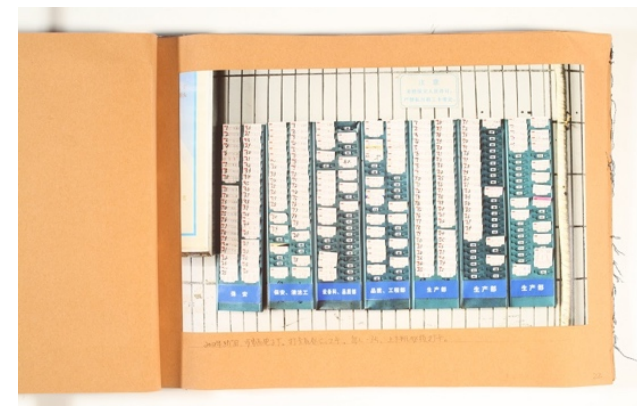

\section{Catalogue 24}

Collection: Lavis Fine Arts

Index No.: 30427_30-24

Title: Bubu Gao Electronics Factory. Cards at the Punch Card Machine. One Card per Person: Employees Are Required to Punch in and out Before and After Work.

Artist: Zhan Youbing

Location: 65QSW21 - 65 Queen Street West, 21 ${ }^{\text {st }}$ floor

Category: Albums

Medium: D2T2 print mounted on kraft paper

Condition: Excellent, minor cockling

Date of Origin: 7 August 2012

Place of Origin: Dongguan, Guangdong, China

Description: None

Size: Image: $34.5 \times 21.8 \mathrm{~cm}(13.58 \times 8.58$ inches $)$

Mount: $42 \times 29.6 \mathrm{~cm}(16.53 \times 11.65$ inches $)$

Signature/Inscriptions: Titled and dated, in pencil, au mount recto

Purchased From: WassinkLundgren (Ruben Lundgren)

Date of Acquisition: 20 October 2016

AMC No.: AMC 16525 


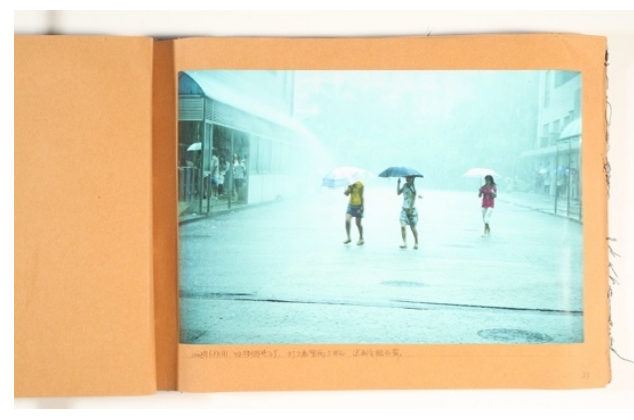

\section{Catalogue 25}

Collection: Lavis Fine Arts

Index No.: 30427_30-25

Title: Shili Technology and Electronics Factory. Migrant Workers Rushing to Work in the Rain. They Will Be Penalized for Lateness.

Artist: Zhan Youbing

Location: 65QSW21 - 65 Queen Street West, 21 ${ }^{\text {st }}$ floor

Category: Albums

Medium: D2T2 print mounted on kraft paper

Condition: Excellent, minor cockling

Date of Origin: 13 June 2008

Place of Origin: Dongguan, Guangdong, China

Description: None

Size: Image: $34.4 \times 22.7 \mathrm{~cm}(13.54 \times 8.94$ inches $)$

Mount: $42 \times 29.6 \mathrm{~cm}$ (16.53 x 11.65 inches)

Signature/Inscriptions: Titled and dated, in pencil, au mount recto

Purchased From: WassinkLundgren (Ruben Lundgren)

Date of Acquisition: 20 October 2016

AMC No.: AMC 16525 


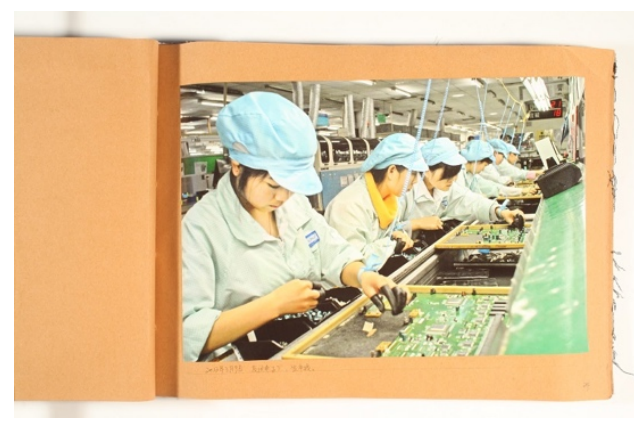

\section{Catalogue 26}

Collection: Lavis Fine Arts

Index No.: 30427_30-26

Title: Youxun Electronics Factory. Production Line.

Artist: Zhan Youbing

Location: 65QSW21 - 65 Queen Street West, 21 ${ }^{\text {st }}$ floor

Category: Albums

Medium: D2T2 print mounted on kraft paper

Condition: Excellent, minor cockling

Date of Origin: 9 March 2012

Place of Origin: Dongguan, Guangdong, China

Description: None

Size: Image: $34.4 \times 23 \mathrm{~cm}(13.54 \times 9.05$ inches $)$

Mount: $42 \times 29.6 \mathrm{~cm}$ (16.53 x 11.65 inches $)$

Signature/Inscriptions: Titled and dated, in pencil, au mount recto

Purchased From: WassinkLundgren (Ruben Lundgren)

Date of Acquisition: 20 October 2016

AMC No.: AMC 16525 


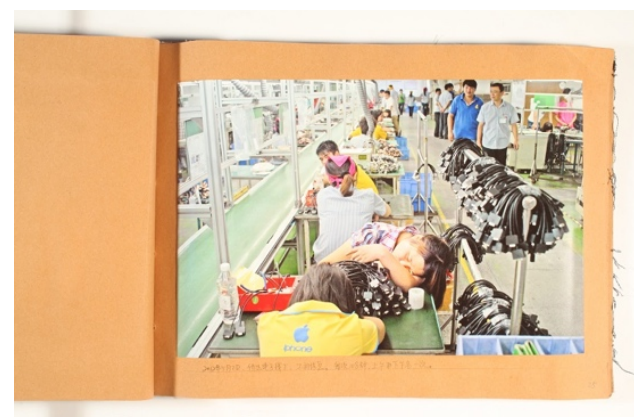

\section{Catalogue 27}

Collection: Lavis Fine Arts

Index No.: 30427_30-27

Title: Qiaoda Electronic Cable Factory. Workshop Break: Two Ten-Minute Breaks, One in the Morning and One in the Afternoon.

Artist: Zhan Youbing

Location: 65QSW21 - 65 Queen Street West, 21 ${ }^{\text {st }}$ floor

Category: Albums

Medium: D2T2 print mounted on kraft paper

Condition: Excellent, minor cockling

Date of Origin: 2 May 2012

Place of Origin: Dongguan, Guangdong, China

Description: None

Size: Image: $34.5 \times 22.8 \mathrm{~cm}(13.58 \times 8.98$ inches $)$ Mount: $42 \times 29.6 \mathrm{~cm}$ (16.53 x 11.65 inches)

Signature/Inscriptions: Titled and dated, in pencil, au mount recto

Purchased From: WassinkLundgren (Ruben Lundgren)

Date of Acquisition: 20 October 2016

AMC No.: AMC 16525 


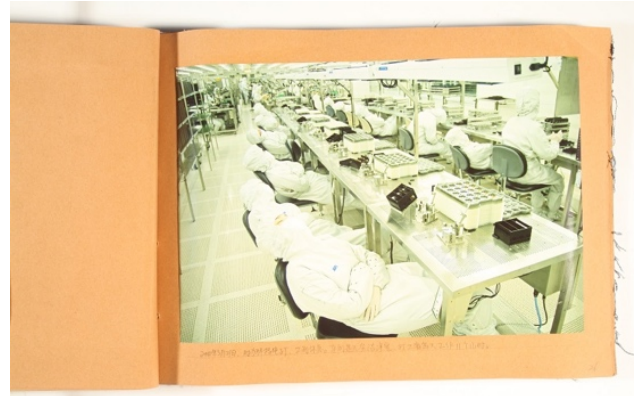

\section{Catalogue 28}

Collection: Lavis Fine Arts

Index No.: 30427_30-28

Title: Shili Technology and Electronics Factory. Workshop Break: Migrant Workers Are Required to Work Eleven Hours a Day in the Dust-Free Cleanroom.

Artist: Zhan Youbing

Location: 65QSW21 - 65 Queen Street West, 21 ${ }^{\text {st }}$ floor

Category: Albums

Medium: D2T2 print mounted on kraft paper

Condition: Excellent, minor cockling

Date of Origin: 21 May 2010

Place of Origin: Dongguan, Guangdong, China

Description: None

Size: Image: $34.3 \times 23 \mathrm{~cm}(13.50 \times 9.05$ inches $)$

Mount: $42 \times 29.6 \mathrm{~cm}$ (16.53 x 11.65 inches)

Signature/Inscriptions: Titled and dated, in pencil, au mount recto

Purchased From: WassinkLundgren (Ruben Lundgren)

Date of Acquisition: 20 October 2016

AMC No.: AMC 16525 


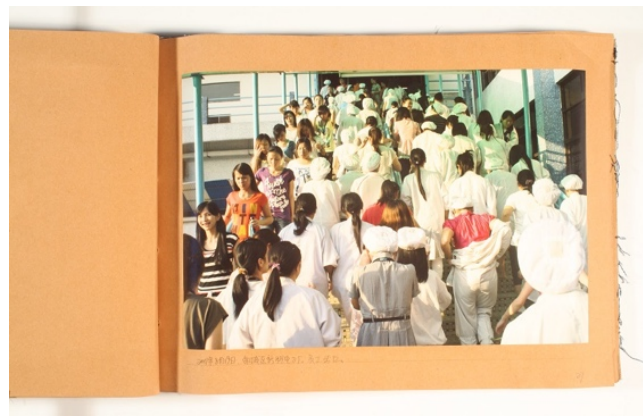

\section{Catalogue 29}

Collection: Lavis Fine Arts

Index No.: 30427_30-29

Title: Nancheng District Xinke Electronics Factory. Exit.

Artist: Zhan Youbing

Location: 65QSW21 - 65 Queen Street West, 21 ${ }^{\text {st }}$ floor

Category: Albums

Medium: D2T2 print mounted on kraft paper

Condition: Excellent, minor cockling

Date of Origin: 19 August 2011

Place of Origin: Dongguan, Guangdong, China

Description: None

Size: Image: $34.2 \times 22.8 \mathrm{~cm}(13.46 \times 8.98$ inches $)$

Mount: $42 \times 29.6 \mathrm{~cm}(16.53 \times 11.65$ inches $)$

Signature/Inscriptions: Titled and dated, in pencil, au mount recto

Purchased From: WassinkLundgren (Ruben Lundgren)

Date of Acquisition: 20 October 2016

AMC No.: AMC 16525 


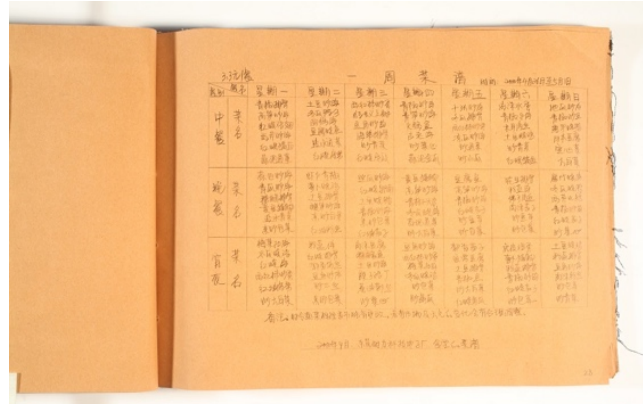

\section{Catalogue 30}

Collection: Lavis Fine Arts

Index No.: 30427_30-30

Title: Dongguan Shili Technology and Electronics Factory. Canteen Menu.

Artist: Zhan Youbing

Location: 65QSW21 - 65 Queen Street West, 21 ${ }^{\text {st }}$ floor

Category: Albums

Medium: Kraft paper

Condition: Excellent

Date of Origin: April 2010

Place of Origin: Dongguan, Guangdong, China

Description: Canteen menu copied in Chinese by Zhan

Size: 42 × $29.6 \mathrm{~cm}$ (16.53 x 11.65 inches)

Signature/Inscriptions: Titled and dated, in pencil, au mount recto

Purchased From: WassinkLundgren (Ruben Lundgren)

Date of Acquisition: 20 October 2016

AMC No.: AMC 16525 


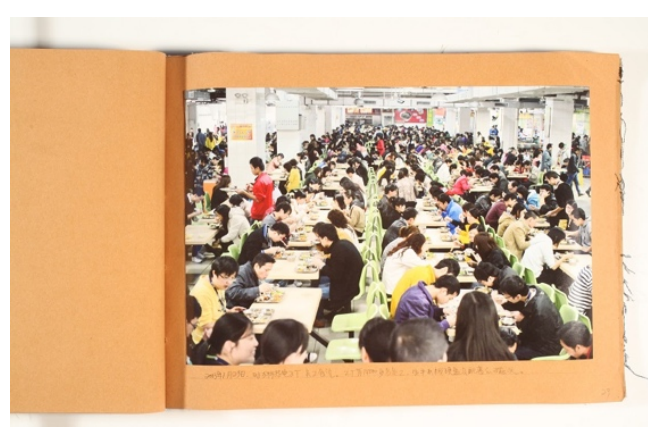

\section{Catalogue 31}

Collection: Lavis Fine Arts

Index No.: 30427_30-31

Title: Shili Technology and Electronics Factory. Canteen. More Than Fourteen Thousand Employees Manufacture Mechanical Hard Drives and Supplementary Magnetic Heads in the Factory.

Artist: Zhan Youbing

Location: 65QSW21 - 65 Queen Street West, 21 ${ }^{\text {st }}$ floor

Category: Albums

Medium: D2T2 print mounted on kraft paper

Condition: Excellent, minor cockling

Date of Origin: 29 January 2013

Place of Origin: Dongguan, Guangdong, China

Description: None

Size: Image: $34.3 \times 23 \mathrm{~cm}(13.50 \times 9.05$ inches $)$

Mount: 42 x $29.6 \mathrm{~cm}(16.53 \times 11.65$ inches $)$

Signature/Inscriptions: Titled and dated, in pencil, au mount recto

Purchased From: WassinkLundgren (Ruben Lundgren)

Date of Acquisition: 20 October 2016

AMC No.: AMC 16525 


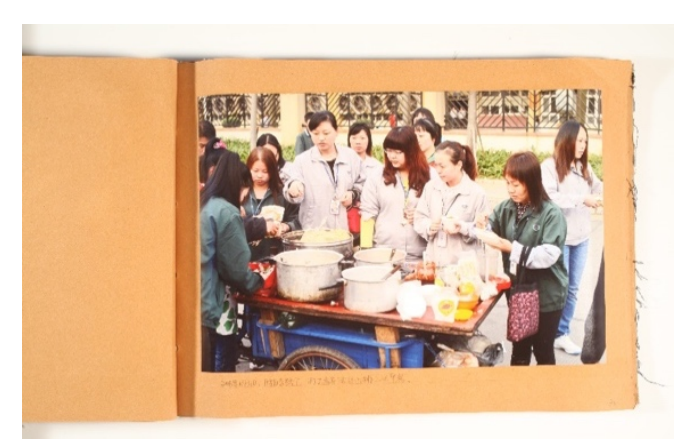

\section{Catalogue 32}

Collection: Lavis Fine Arts

Index No.: 30427_30-32

Title: Sheng Baiji Shoe Factory. Migrant Workers Buying Breakfast at the Hawker Stall.

Artist: Zhan Youbing

Location: 65QSW21 - 65 Queen Street West, 21 ${ }^{\text {st }}$ floor

Category: Albums

Medium: D2T2 print mounted on kraft paper

Condition: Excellent, minor cockling

Date of Origin: 29 January 2013

Place of Origin: Dongguan, Guangdong, China

Description: None

Size: Image: $34.3 \times 23 \mathrm{~cm}(13.50 \times 9.05$ inches $)$

Mount: $42 \times 29.6 \mathrm{~cm}$ (16.53 x 11.65 inches)

Signature/Inscriptions: Titled and dated, in pencil, au mount recto

Purchased From: WassinkLundgren (Ruben Lundgren)

Date of Acquisition: 20 October 2016

AMC No.: AMC 16525 


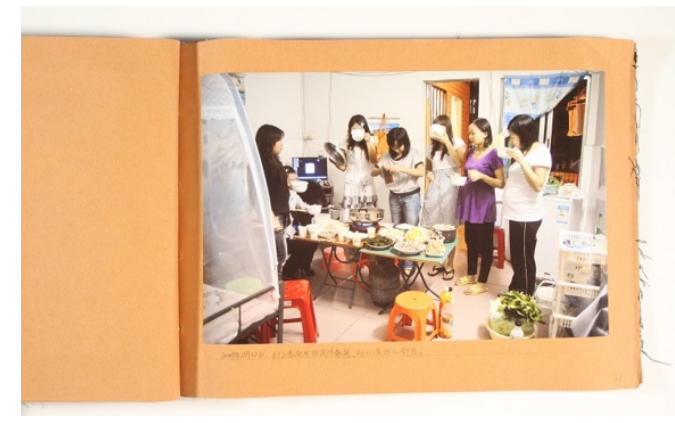

\section{Catalogue 33}

Collection: Lavis Fine Arts

Index No.: 30427_30-33

Title: Migrant Workers Gathering for Dinner in the Rental Apartment. "They Are My Neighbors."

Artist: Zhan Youbing

Location: 65QSW21 - 65 Queen Street West, 21 ${ }^{\text {st }}$ floor

Category: Albums

Medium: D2T2 print mounted on kraft paper

Condition: Excellent, minor cockling

Date of Origin: 12 November 2009

Place of Origin: Dongguan, Guangdong, China

Description: None

Size: Image: $34.4 \times 22.8 \mathrm{~cm}(13.54 \times 8.98$ inches $)$

Mount: $42 \times 29.6 \mathrm{~cm}$ (16.53 x 11.65 inches)

Signature/Inscriptions: Titled and dated, in pencil, au mount recto

Purchased From: WassinkLundgren (Ruben Lundgren)

Date of Acquisition: 20 October 2016

AMC No.: AMC 16525 


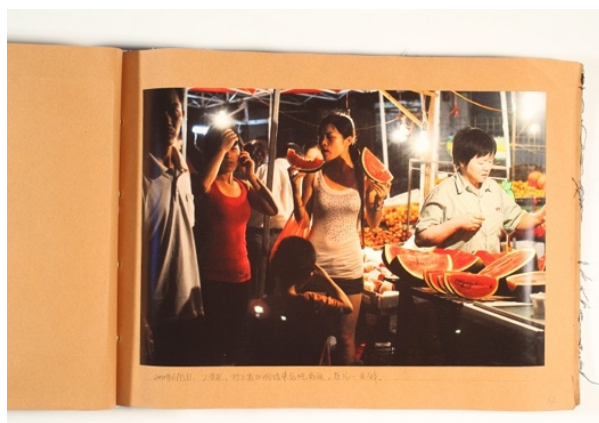

\section{Catalogue 34}

Collection: Lavis Fine Arts

Index No.: 30427_30-34

Title: Industrial District. Migrant Workers Eating Watermelon After Working Overtime: Each Piece Costs One Yuan.

Artist: Zhan Youbing

Location: 65QSW21 - 65 Queen Street West, 21 ${ }^{\text {st }}$ floor

Category: Albums

Medium: D2T2 print mounted on kraft paper

Condition: Excellent, minor cockling

Date of Origin: 5 June 2010

Place of Origin: Dongguan, Guangdong, China

Description: 1 yuan $=0.2 \mathrm{CAD}$

Size: Image: $34.2 \times 23 \mathrm{~cm}(13.46 \times 9.05$ inches $)$

Mount: $42 \times 29.6 \mathrm{~cm}(16.53 \times 11.65$ inches $)$

Signature/Inscriptions: Titled and dated, in pencil, au mount recto

Purchased From: WassinkLundgren (Ruben Lundgren)

Date of Acquisition: 20 October 2016

AMC No.: AMC 16525 


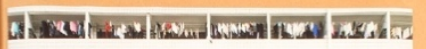

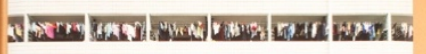

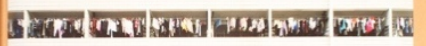

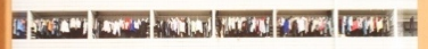

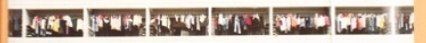

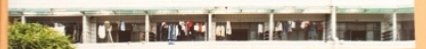

\section{Catalogue 35}

Collection: Lavis Fine Arts

Index No.: 30427_30-35

Title: Nancheng District Xinke Electronics Factory. Dormitory. The Floor Area of Each Room Is about Twenty Square Meters and Each Room Accommodates

Ten People.

Artist: Zhan Youbing

Location: 65QSW21 - 65 Queen Street West, 21 ${ }^{\text {st }}$ floor

Category: Albums

Medium: D2T2 print mounted on kraft paper

Condition: Excellent, minor cockling

Date of Origin: 12 January 2010

Place of Origin: Dongguan, Guangdong, China

Description: None

Size: Image: $34.5 \times 25 \mathrm{~cm}(13.58 \times 9.84$ inches $)$

Mount: $42 \times 29.6 \mathrm{~cm}$ (16.53 x 11.65 inches)

Signature/Inscriptions: Titled and dated, in pencil, au mount recto

Purchased From: WassinkLundgren (Ruben Lundgren)

Date of Acquisition: 20 October 2016

AMC No.: AMC 16525 


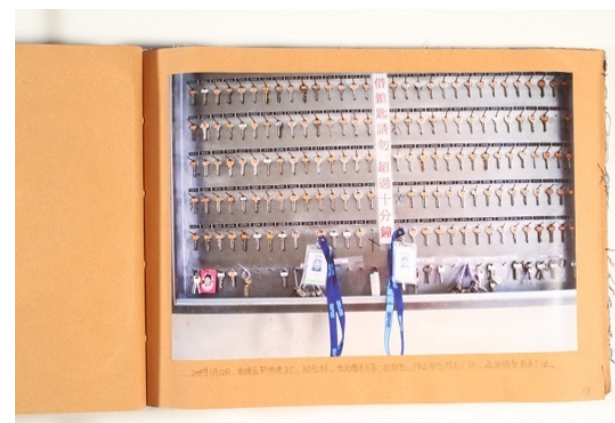

\section{Catalogue 36}

Collection: Lavis Fine Arts

Index No.: 30427_30-36

Title: Nancheng District Xinke Electronics Factory. Dormitory. Key Cabinet. Only One Key to Each Room: Anyone Who Borrows a Key Has to Hang up His/Her Staff Card Which He/She Will Get Back When the Key Is Returned.

Artist: Zhan Youbing

Location: 65QSW21 - 65 Queen Street West, 21 ${ }^{\text {st }}$ floor

Category: Albums

Medium: D2T2 print mounted on kraft paper

Condition: Excellent, minor cockling

Date of Origin: 12 January 2010

Place of Origin: Dongguan, Guangdong, China

Description: None

Size: Image: $34.2 \times 23.5 \mathrm{~cm}(13.46 \times 9.25$ inches $)$

Mount: $42 \times 29.6 \mathrm{~cm}$ (16.53 x 11.65 inches)

Signature/Inscriptions: Titled and dated, in pencil, au mount recto

Purchased From: WassinkLundgren (Ruben Lundgren)

Date of Acquisition: 20 October 2016

AMC No.: AMC 16525 


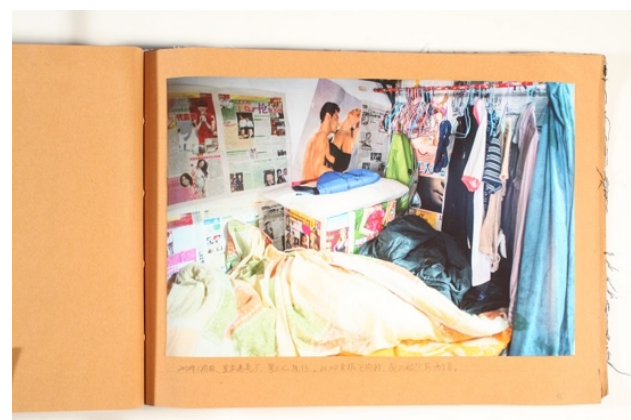

\section{Catalogue 37}

Collection: Lavis Fine Arts

Index No.: 30427_30-37

Title: Meigao Watchcase Factory. Male Worker's Bed. Owner of Factory Has Absconded, Employees Owed Wages for Two Months.

Artist: Zhan Youbing

Location: 65QSW21 - 65 Queen Street West, 21 ${ }^{\text {st }}$ floor

Category: Albums

Medium: D2T2 print mounted on kraft paper

Condition: Excellent, minor cockling

Date of Origin: 1 December 2012

Place of Origin: Dongguan, Guangdong, China

Description: None

Size: Image: $34.5 \times 23 \mathrm{~cm}(13.58 \times 9.05$ inches $)$

Mount: 42 × $29.6 \mathrm{~cm}$ (16.53 x 11.65 inches)

Signature/Inscriptions: Titled and dated, in pencil, au mount recto

Purchased From: WassinkLundgren (Ruben Lundgren)

Date of Acquisition: 20 October 2016

AMC No.: AMC 16525 


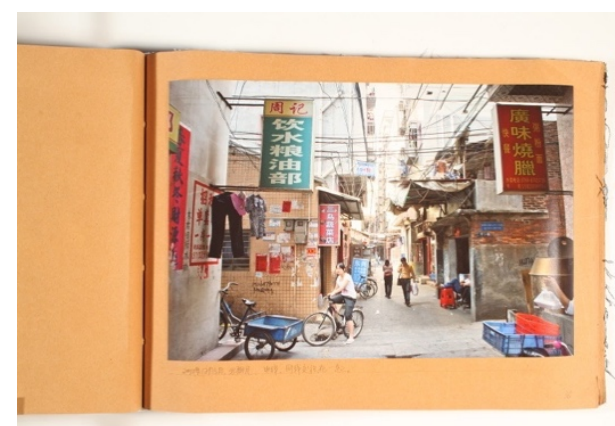

\section{Catalogue 38}

Collection: Lavis Fine Arts

Index No.: 30427_30-38

Title: Rental Apartments. Intertwined Wires and Network Cables.

Artist: Zhan Youbing

Location: 65QSW21 - 65 Queen Street West, 21 ${ }^{\text {st }}$ floor

Category: Albums

Medium: D2T2 print mounted on kraft paper

Condition: Excellent, minor cockling

Date of Origin: 5 December 2012

Place of Origin: Dongguan, Guangdong, China

Description: None

Size: Image: $34.4 \times 23 \mathrm{~cm}(13.54 \times 9.05$ inches $)$

Mount: $42 \times 29.6 \mathrm{~cm}$ (16.53 x 11.65 inches)

Signature/Inscriptions: Titled and dated, in pencil, au mount recto

Purchased From: WassinkLundgren (Ruben Lundgren)

Date of Acquisition: 20 October 2016

AMC No.: AMC 16525 


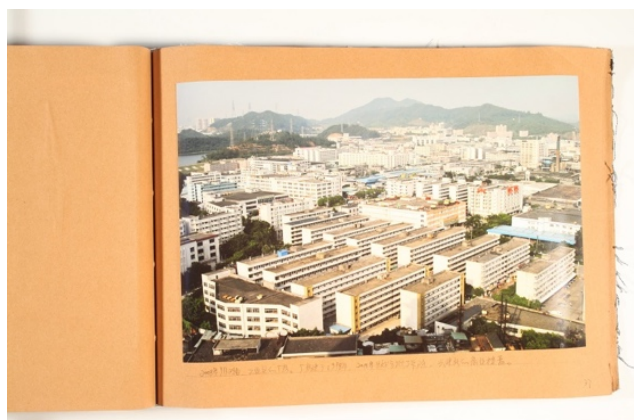

\section{Catalogue 39}

Collection: Lavis Fine Arts

Index No.: 30427_30-39

Title: Factories in Industrial District. Factories Were Built in 1998. In 2010, They Were Demolished in Batches and New Commercial Real Estate Was Built.

Artist: Zhan Youbing

Location: 65QSW21 - 65 Queen Street West, 21 ${ }^{\text {st }}$ floor

Category: Albums

Medium: D2T2 print mounted on kraft paper

Condition: Excellent, minor cockling

Date of Origin: 28 September 2008

Place of Origin: Dongguan, Guangdong, China

Description: None

Size: Image: $34 \times 23 \mathrm{~cm}(13.39 \times 9.05$ inches $)$

Mount: $42 \times 29.6 \mathrm{~cm}$ (16.53 x 11.65 inches)

Signature/Inscriptions: Titled and dated, in pencil, au mount recto

Purchased From: WassinkLundgren (Ruben Lundgren)

Date of Acquisition: 20 October 2016

AMC No.: AMC 16525 


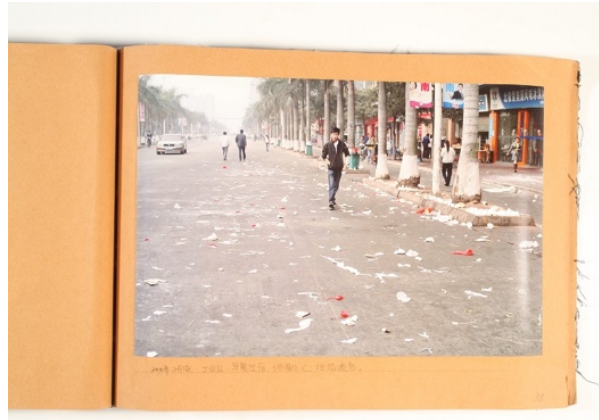

\section{Catalogue 40}

Collection: Lavis Fine Arts

Index No.: 30427_30-

Title: Industrial District. Litter Everywhere After Breakfast.

Artist: Zhan Youbing

Location: 65QSW21 - 65 Queen Street West, 21 ${ }^{\text {st }}$ floor

Category: Albums

Medium: D2T2 print mounted on kraft paper

Condition: Excellent, minor cockling

Date of Origin: 1 February 2010

Place of Origin: Dongguan, Guangdong, China

Description: None

Size: Image: 34.4 × $22.6 \mathrm{~cm}(13.54 \times 8.90$ inches $)$

Mount: $42 \times 29.6 \mathrm{~cm}(16.53 \times 11.65$ inches $)$

Signature/Inscriptions: Titled and dated, in pencil, au mount recto

Purchased From: WassinkLundgren (Ruben Lundgren)

Date of Acquisition: 20 October 2016

AMC No.: AMC 16525 


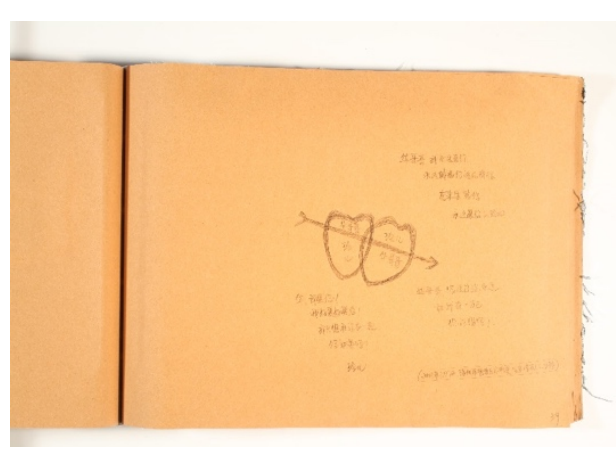

\section{Catalogue 41}

Collection: Lavis Fine Arts

Index No.: 30427_30-41

Title: Graffiti on Pavilion, Gong Ming De Park, Baoan District, Shenzhen.

Artist: Zhan Youbing

Location: 65QSW21 - 65 Queen Street West, 21 ${ }^{\text {st }}$ floor

Category: Albums

Medium: Kraft paper

Condition: Excellent

Date of Origin: 7 March 2013

Place of Origin: Dongguan, Guangdong, China

Description: Graffiti written on pavilion by migrant workers, copied by Zhan

Size: 42 x $29.6 \mathrm{~cm}$ (16.53 x 11.65 inches)

Signature/Inscriptions: Titled and dated, in pencil

Purchased From: WassinkLundgren (Ruben Lundgren)

Date of Acquisition: 20 October 2016

AMC No.: AMC 16525 


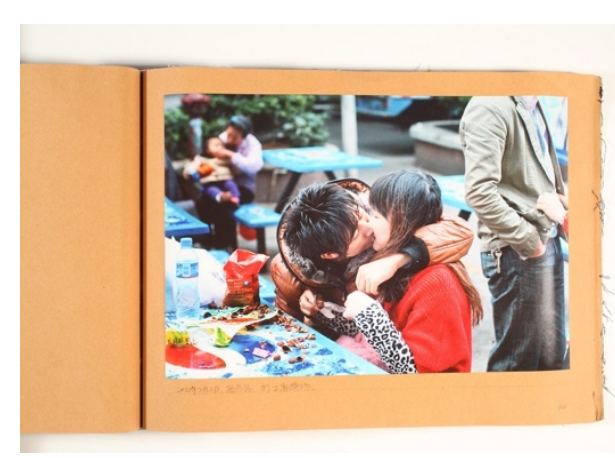

\section{Catalogue 42}

Collection: Lavis Fine Arts

Index No.: 30427_30-42

Title: Migrant Workers Kissing Outside Supermarket.

Artist: Zhan Youbing

Location: 65QSW21 - 65 Queen Street West, 21 ${ }^{\text {st }}$ floor

Category: Albums

Medium: D2T2 print mounted on kraft paper

Condition: Excellent, minor cockling

Date of Origin: 11 February 2012

Place of Origin: Dongguan, Guangdong, China

Description: None

Size: Image: $34.1 \times 23 \mathrm{~cm}(13.43 \times 9.05$ inches $)$

Mount: $42 \times 29.6 \mathrm{~cm}$ (16.53 x 11.65 inches)

Signature/Inscriptions: Titled and dated, in pencil, au mount recto

Purchased From: WassinkLundgren (Ruben Lundgren)

Date of Acquisition: 20 October 2016

AMC No.: AMC 16525 


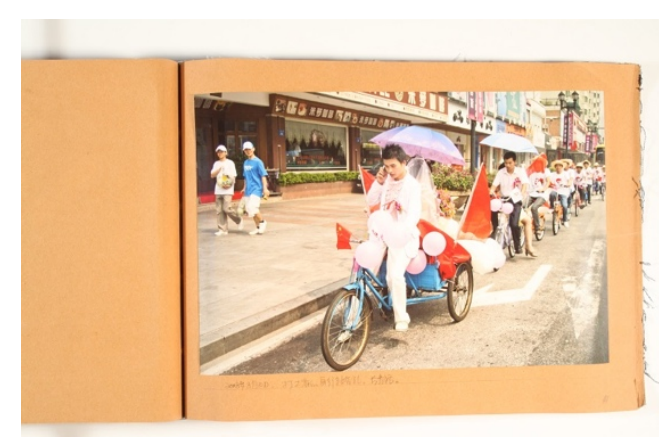

\section{Catalogue 43}

Collection: Lavis Fine Arts

Index No.: 30427_30-43

Title: Bicycle Wedding of Migrant Workers. Changqing Road.

Artist: Zhan Youbing

Location: 65QSW21 - 65 Queen Street West, 21 ${ }^{\text {st }}$ floor

Category: Albums

Medium: D2T2 print mounted on kraft paper

Condition: Excellent, minor cockling

Date of Origin: 10 August 2008

Place of Origin: Dongguan, Guangdong, China

Description: None

Size: Image: $34.5 \times 23.1 \mathrm{~cm}(13.58 \times 9.09$ inches $)$ Mount: 42 x $29.6 \mathrm{~cm}$ (16.53 x 11.65 inches)

Signature/Inscriptions: Titled and dated, in pencil, au mount recto

Purchased From: WassinkLundgren (Ruben Lundgren)

Date of Acquisition: 20 October 2016

AMC No.: AMC 16525 


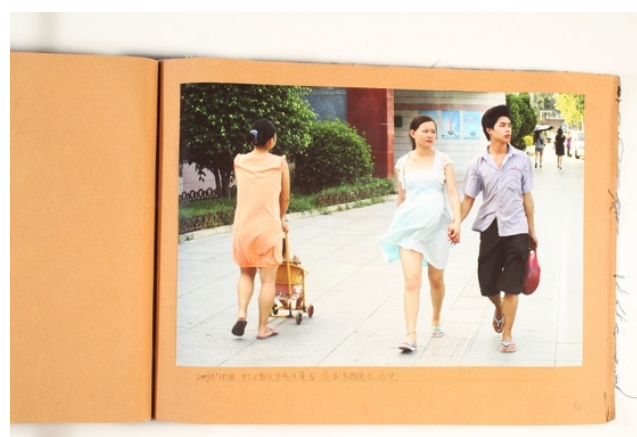

\section{Catalogue 44}

Collection: Lavis Fine Arts

Index No.: 30427_30-44

Title: Migrant Workers on Their Way to Rental Apartment After Grocery Shopping at Supermarket.

Artist: Zhan Youbing

Location: 65QSW21 - 65 Queen Street West, 21 ${ }^{\text {st }}$ floor

Category: Albums

Medium: D2T2 print mounted on kraft paper

Condition: Excellent, minor cockling

Date of Origin: 1 July 2009

Place of Origin: Dongguan, Guangdong, China

Description: None

Size: Image: $34.2 \times 23 \mathrm{~cm}(13.46 \times 9.05$ inches $)$

Mount: $42 \times 29.6 \mathrm{~cm}(16.53 \times 11.65$ inches $)$

Signature/Inscriptions: Titled and dated, in pencil, au mount recto

Purchased From: WassinkLundgren (Ruben Lundgren)

Date of Acquisition: 20 October 2016

AMC No.: AMC 16525 


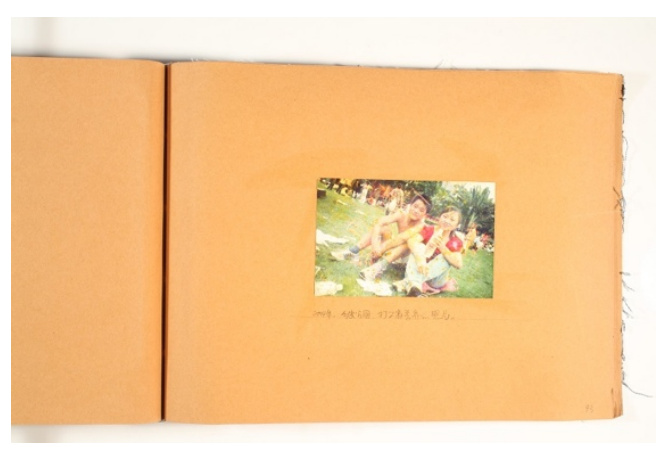

\section{Catalogue 45}

Collection: Lavis Fine Arts

Index No.: 30427_30-45

Title: Changan Park. Photograph Discarded by Migrant Workers.

Artist: Unknown

Location: 65QSW21 - 65 Queen Street West, 21 ${ }^{\text {st }}$ floor

Category: Albums

Medium: D2T2 print mounted on kraft paper

Condition: Major creases, cockling and discoloration

Date of Origin: 2014

Place of Origin: Dongguan, Guangdong, China

Description: Photograph found by Zhan Youbing

Size: Image: 14.9 x $9.7 \mathrm{~cm}(5.87 \times 3.82$ inches $)$

Mount: $42 \times 29.6 \mathrm{~cm}$ (16.53 x 11.65 inches $)$

Signature/Inscriptions: Titled and dated, in pencil, au mount recto

Purchased From: WassinkLundgren (Ruben Lundgren)

Date of Acquisition: 20 October 2016

AMC No.: AMC 16525 


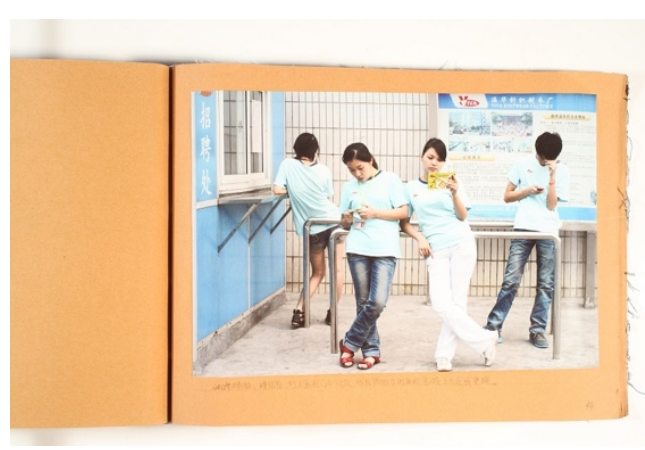

\section{Catalogue 46}

Collection: Lavis Fine Arts

Index No.: 30427_30-46

Title: Migrant Workers Relaxing at the Front Gate after Dinner. They Have to Work Overtime Until at Least 9pm.

Artist: Zhan Youbing

Location: 65QSW21 - 65 Queen Street West, 21 ${ }^{\text {st }}$ floor

Category: Albums

Medium: D2T2 print mounted on kraft paper

Condition: Excellent, minor cockling

Date of Origin: 13 October 2012

Place of Origin: Dongguan, Guangdong, China

Description: None

Size: Image: $34.5 \times 23.3 \mathrm{~cm}(13.58 \times 9.17$ inches $)$

Mount: $42 \times 29.6 \mathrm{~cm}(16.53 \times 11.65$ inches $)$

Signature/Inscriptions: Titled and dated, in pencil, au mount recto

Purchased From: WassinkLundgren (Ruben Lundgren)

Date of Acquisition: 20 October 2016

AMC No.: AMC 16525 


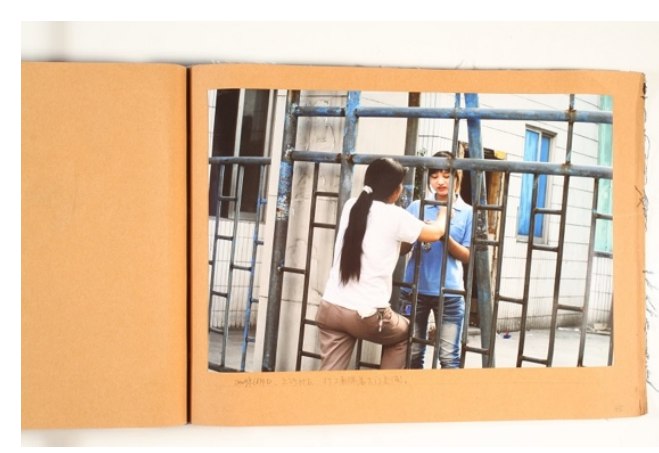

\section{Catalogue 47}

Collection: Lavis Fine Arts

Index No.: 30427_30-47

Title: Shangsha Community. Migrant Workers Communicating Through the Gate.

Artist: Zhan Youbing

Location: 65QSW21 - 65 Queen Street West, 21 ${ }^{\text {st }}$ floor

Category: Albums

Medium: D2T2 print mounted on kraft paper

Condition: Excellent, minor cockling

Date of Origin: 4 June 2009

Place of Origin: Dongguan, Guangdong, China

Description: None

Size: Image: $34.4 \times 23 \mathrm{~cm}(13.54 \times 9.05$ inches $)$

Mount: $42 \times 29.6 \mathrm{~cm}(16.53 \times 11.65$ inches $)$

Signature/Inscriptions: Titled and dated, in pencil, au mount recto

Purchased From: WassinkLundgren (Ruben Lundgren)

Date of Acquisition: 20 October 2016

AMC No.: AMC 16525 


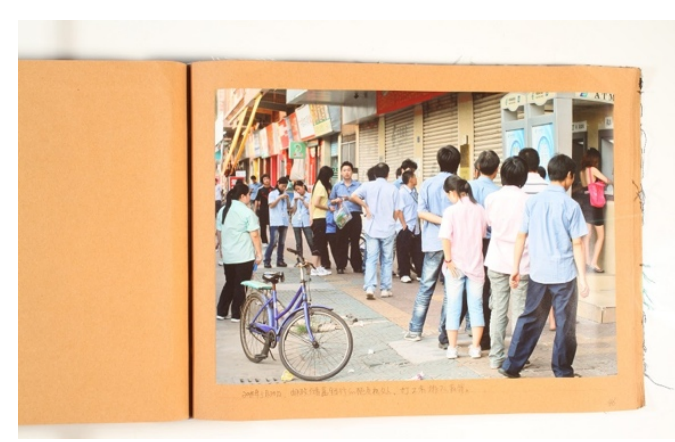

\section{Catalogue 48}

Collection: Lavis Fine Arts

Index No.: 30427_30-48

Title: ATMs of Postal Saving Bank of China. Migrant Workers Lining up to Withdraw Money.

Artist: Zhan Youbing

Location: 65QSW21 - 65 Queen Street West, 21 ${ }^{\text {st }}$ floor

Category: Albums

Medium: D2T2 print mounted on kraft paper

Condition: Excellent, minor cockling

Date of Origin: 20 May 2010

Place of Origin: Dongguan, Guangdong, China

Description: None

Size: Image: $34.5 \times 24.3 \mathrm{~cm}(13.58 \times 9.57$ inches $)$

Mount: $42 \times 29.6 \mathrm{~cm}$ (16.53 x 11.65 inches)

Signature/Inscriptions: Titled and dated, in pencil, au mount recto

Purchased From: WassinkLundgren (Ruben Lundgren)

Date of Acquisition: 20 October 2016

AMC No.: AMC 16525 


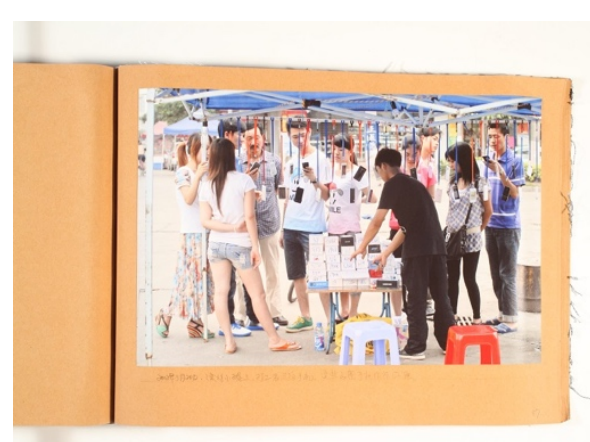

\section{Catalogue 49}

Collection: Lavis Fine Arts

Index No.: 30427_30-49

Title: Migrant Workers Purchasing Cellphones at the Hawker Stall. Price of These Knockoff Cellphones Is Low.

Artist: Zhan Youbing

Location: 65QSW21 - 65 Queen Street West, 21 ${ }^{\text {st }}$ floor

Category: Albums

Medium: D2T2 print mounted on kraft paper

Condition: Excellent, minor cockling

Date of Origin: 20 May 2012

Place of Origin: Dongguan, Guangdong, China

Description: None

Size: Image: $34.3 \times 22.8 \mathrm{~cm}(13.50 \times 8.98$ inches $)$

Mount: $42 \times 29.6 \mathrm{~cm}(16.53 \times 11.65$ inches $)$

Signature/Inscriptions: Titled and dated, in pencil, au mount recto

Purchased From: WassinkLundgren (Ruben Lundgren)

Date of Acquisition: 20 October 2016

AMC No.: AMC 16525 


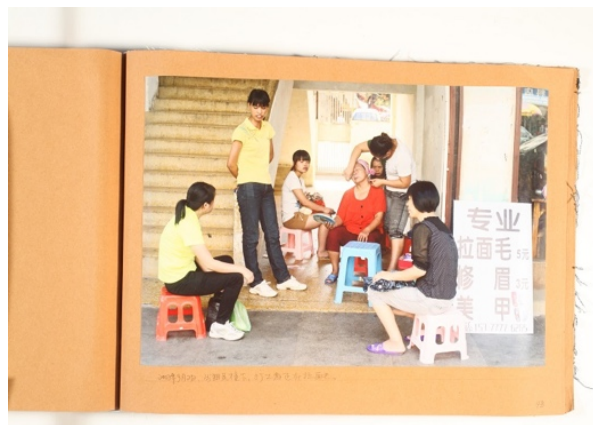

\section{Catalogue 50}

Collection: Lavis Fine Arts

Index No.: 30427_30-50

Title: Downstairs of Rental Apartments. Migrant Workers Having Facial Hair Removed.

Artist: Zhan Youbing

Location: 65QSW21 - 65 Queen Street West, 21 ${ }^{\text {st }}$ floor

Category: Albums

Medium: D2T2 print mounted on kraft paper

Condition: Excellent, minor cockling

Date of Origin: 2 September 2012

Place of Origin: Dongguan, Guangdong, China

Description: None

Size: Image: $34.4 \times 23.6 \mathrm{~cm}(13.54 \times 9.29$ inches $)$ Mount: $42 \times 29.6 \mathrm{~cm}$ (16.53 x 11.65 inches)

Signature/Inscriptions: Titled and dated, in pencil, au mount recto

Purchased From: WassinkLundgren (Ruben Lundgren)

Date of Acquisition: 20 October 2016

AMC No.: AMC 16525 


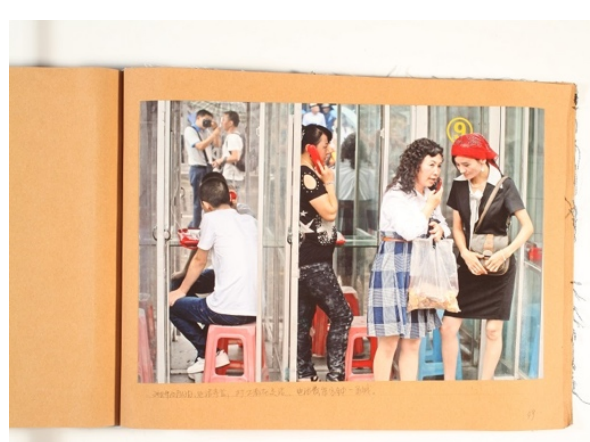

\section{Catalogue 51}

Collection: Lavis Fine Arts

Index No.: 30427_30-51

Title: Migrant Workers Chatting in Front of Telephone Booths. Telephone Fee Is 0.1 Yuan per Minute.

Artist: Zhan Youbing

Location: 65QSW21 - 65 Queen Street West, 21 ${ }^{\text {st }}$ floor

Category: Albums

Medium: D2T2 print mounted on kraft paper

Condition: Excellent, minor cockling

Date of Origin: 12 October 2011

Place of Origin: Dongguan, Guangdong, China

Description: 0.1 yuan $=0.02 \mathrm{CAD}$

Size: Image: $34.5 \times 23 \mathrm{~cm}(13.58 \times 9.05$ inches $)$

Mount: $42 \times 29.6 \mathrm{~cm}(16.53 \times 11.65$ inches $)$

Signature/Inscriptions: Titled and dated, in pencil, au mount recto

Purchased From: WassinkLundgren (Ruben Lundgren)

Date of Acquisition: 20 October 2016

AMC No.: AMC 16525 


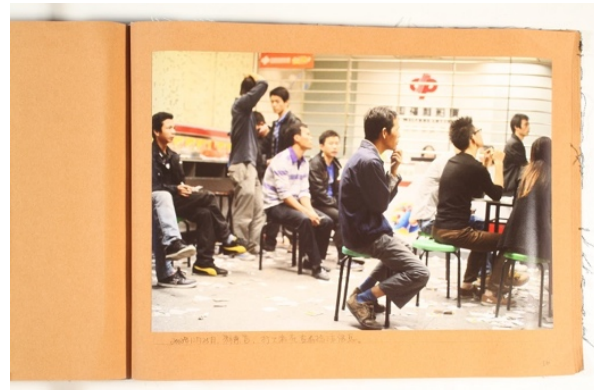

\section{Catalogue 52}

Collection: Lavis Fine Arts

Index No.: 30427_30-52

Title: Lottery Shop. Migrant Workers Reviewing Betting Information.

Artist: Zhan Youbing

Location: 65QSW21 - 65 Queen Street West, 21 ${ }^{\text {st }}$ floor

Category: Albums

Medium: D2T2 print mounted on kraft paper

Condition: Excellent, minor cockling

Date of Origin: 25 November 2012

Place of Origin: Dongguan, Guangdong, China

Description: None

Size: Image: $34.3 \times 23.2 \mathrm{~cm}(13.50 \times 9.13$ inches $)$

Mount: 42 × $29.6 \mathrm{~cm}(16.53 \times 11.65$ inches $)$

Signature/Inscriptions: Titled and dated, in pencil, au mount recto

Purchased From: WassinkLundgren (Ruben Lundgren)

Date of Acquisition: 20 October 2016

AMC No.: AMC 16525 


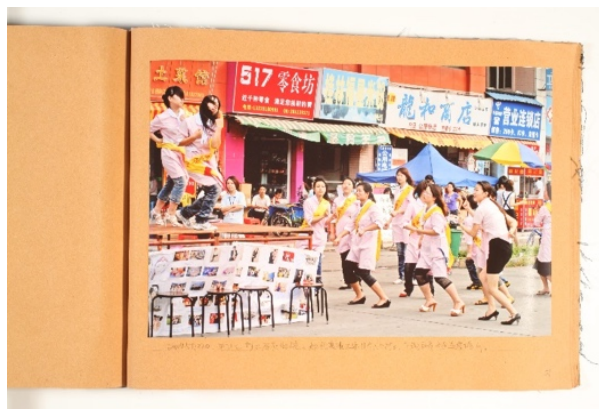

\section{Catalogue 53}

Collection: Lavis Fine Arts

Index No.: 30427_30-53

Title: Migrant Workers of Electronics Factory Doing Exercises. After the ElevenHour Night Shift, They Participate in Beauty Training at Their Own Expense.

Artist: Zhan Youbing

Location: 65QSW21 - 65 Queen Street West, 21 ${ }^{\text {st }}$ floor

Category: Albums

Medium: D2T2 print mounted on kraft paper

Condition: Excellent, minor cockling

Date of Origin: 22 May 2010

Place of Origin: Dongguan, Guangdong, China

Description: None

Size: Image: $34.5 \times 22.9 \mathrm{~cm}(13.58 \times 9.02$ inches $)$

Mount: $42 \times 29.6 \mathrm{~cm}(16.53 \times 11.65$ inches $)$

Signature/Inscriptions: Titled and dated, in pencil, au mount recto

Purchased From: WassinkLundgren (Ruben Lundgren)

Date of Acquisition: 20 October 2016

AMC No.: AMC 16525 


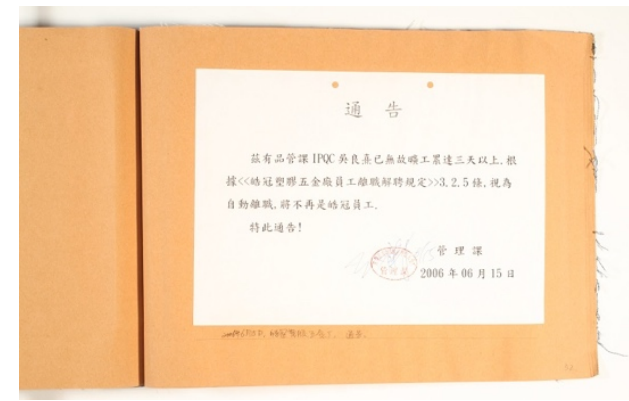

\section{Catalogue 54}

Collection: Lavis Fine Arts

Index No.: 30427_30-54

Title: Haoguan Plastic and Hardware Factory. Layoff Notice.

Artist: Zhan Youbing

Location: 65QSW21 - 65 Queen Street West, 21 ${ }^{\text {st }}$ floor

Category: Albums

Medium: Paper mounted on kraft paper

Condition: Excellent, minor creases in centre left area

Date of Origin: 15 June 2006

Place of Origin: Dongguan, Guangdong, China

Description: None

Size: Image: $29.5 \times 21.1 \mathrm{~cm}(11.61 \times 8.31$ inches $)$

Mount: 42 x $29.6 \mathrm{~cm}$ (16.53 x 11.65 inches)

Signature/Inscriptions: Titled and dated, in pencil, au mount recto

Purchased From: WassinkLundgren (Ruben Lundgren)

Date of Acquisition: 20 October 2016

AMC No.: AMC 16525 


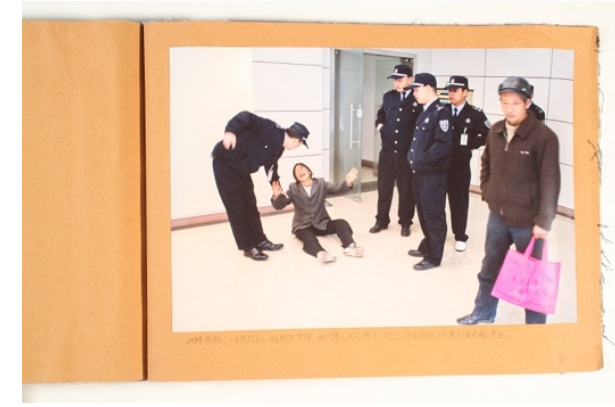

\section{Catalogue 55}

Collection: Lavis Fine Arts

Index No.: 30427_30-55

Title: A Crying Mother from Shanxi. Her 19-Year-Old Daughter Was an

Employee of the Factory: She Drowned in the Sea While She Was on Vacation.

Artist: Zhan Youbing

Location: 65QSW21 - 65 Queen Street West, 21 ${ }^{\text {st }}$ floor

Category: Albums

Medium: D2T2 print mounted on kraft paper

Condition: Excellent, minor cockling

Date of Origin: 14 January 2010

Place of Origin: Dongguan, Guangdong, China

Description: None

Size: Image: $34.4 \times 23.4 \mathrm{~cm}(13.54 \times 9.21$ inches $)$

Mount: $42 \times 29.6 \mathrm{~cm}(16.53 \times 11.65$ inches $)$

Signature/Inscriptions: Titled and dated, in pencil, au mount recto

Purchased From: WassinkLundgren (Ruben Lundgren)

Date of Acquisition: 20 October 2016

AMC No.: AMC 16525 


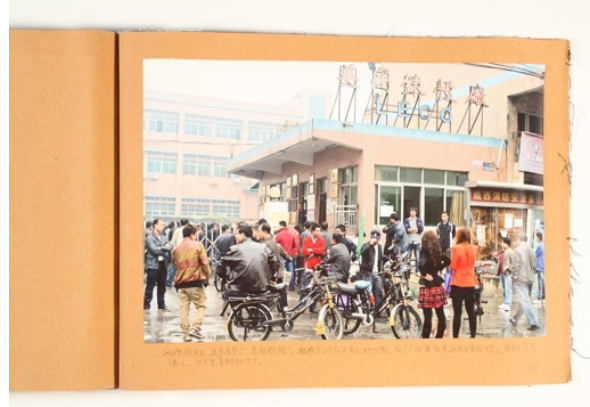

\section{Catalogue 56}

Collection: Lavis Fine Arts

Index No.: 30427_30-56

Title: Meigao Watchcase Factory. The Owner Has Absconded. Owed Wages for Two Months, Migrant Workers Waiting for Relevant Departments to Solve Their Problems. The Owner Is from Hong Kong. Factory Imported Supply Material.

Artist: Zhan Youbing

Location: 65QSW21 - 65 Queen Street West, 21 ${ }^{\text {st }}$ floor

Category: Albums

Medium: D2T2 print mounted on kraft paper

Condition: Excellent, minor cockling

Date of Origin: 30 November 2012

Place of Origin: Dongguan, Guangdong, China

Description: None

Size: Image: $34.3 \times 23.4 \mathrm{~cm}(13.50 \times 9.21$ inches $)$

Mount: $42 \times 29.6 \mathrm{~cm}(16.53 \times 11.65$ inches $)$

Signature/Inscriptions: Titled and dated, in pencil, au mount recto

Purchased From: WassinkLundgren (Ruben Lundgren)

Date of Acquisition: 20 October 2016

AMC No.: AMC 16525 


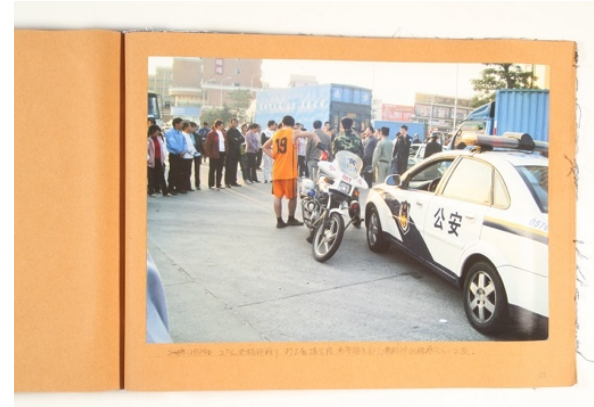

\section{Catalogue 57}

Collection: Lavis Fine Arts

Index No.: 30427_30-57

Title: Factory Owner Has Absconded. Migrant Workers Blocking the Road, Seeking Help from Relevant Departments to Get Back Their Unpaid Wages.

Artist: Zhan Youbing

Location: 65QSW21 - 65 Queen Street West, 21 ${ }^{\text {st }}$ floor

Category: Albums

Medium: D2T2 print mounted on kraft paper

Condition: Excellent, minor cockling

Date of Origin: 24 December 2008

Place of Origin: Dongguan, Guangdong, China

Description: None

Size: Image: $34.3 \times 23.6 \mathrm{~cm}(13.50 \times 9.29$ inches $)$ Mount: 42 x $29.6 \mathrm{~cm}(16.53 \times 11.65$ inches $)$

Signature/Inscriptions: Titled and dated, in pencil, au mount recto

Purchased From: WassinkLundgren (Ruben Lundgren)

Date of Acquisition: 20 October 2016

AMC No.: AMC 16525 


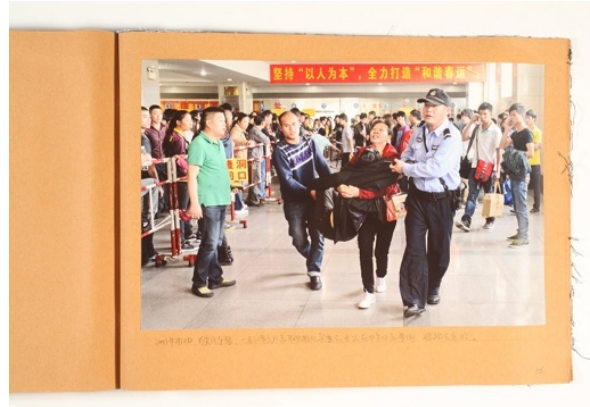

\section{Catalogue 58}

Collection: Lavis Fine Arts

Index No.: 30427_30-58

Title: Changan Bus Station. Female Migrant Worker, Who Had Been Lining up for Tickets from 8am, Fainted at Noon and Was Carried to the Emergency Room.

Artist: Zhan Youbing

Location: 65QSW21 - 65 Queen Street West, 21 ${ }^{\text {st }}$ floor

Category: Albums

Medium: D2T2 print mounted on kraft paper

Condition: Excellent, minor cockling

Date of Origin: 2 February 2013

Place of Origin: Dongguan, Guangdong, China

Description: None

Size: Image: $34.5 \times 22.9 \mathrm{~cm}(13.58 \times 9.02$ inches $)$

Mount: $42 \times 29.6 \mathrm{~cm}(16.53 \times 11.65$ inches $)$

Signature/Inscriptions: Titled and dated, in pencil, au mount recto

Purchased From: WassinkLundgren (Ruben Lundgren)

Date of Acquisition: 20 October 2016

AMC No.: AMC 16525 


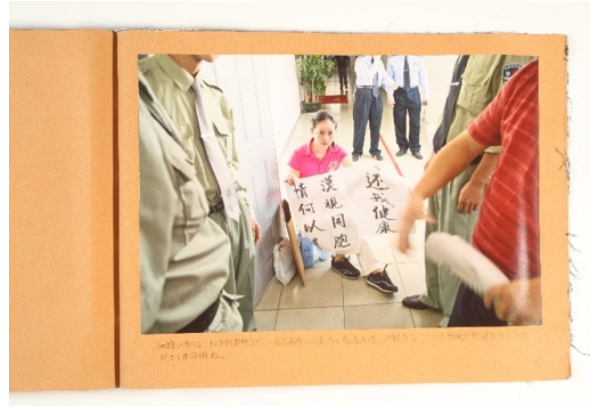

\section{Catalogue 59}

Collection: Lavis Fine Arts

Index No.: 30427_30-59

Title: Shili Technology and Electronics Factory. A Former Cleaner Is Predisposed to Rheumatism: She Suspects the Cause Is That She Had to Work with Cold Water for a Long Period of Time While Working for the Factory.

Artist: Zhan Youbing

Location: 65QSW21 - 65 Queen Street West, 21 ${ }^{\text {st }}$ floor

Category: Albums

Medium: D2T2 print mounted on kraft paper

Condition: Excellent, minor cockling

Date of Origin: 22 October 2008

Place of Origin: Dongguan, Guangdong, China

Description: None

Size: Image: $34.3 \times 23 \mathrm{~cm}(13.50 \times 9.05$ inches $)$

Mount: $42 \times 29.6 \mathrm{~cm}(16.53 \times 11.65$ inches $)$

Signature/Inscriptions: Titled and dated, in pencil, au mount recto

Purchased From: WassinkLundgren (Ruben Lundgren)

Date of Acquisition: 20 October 2016

AMC No.: AMC 16525 


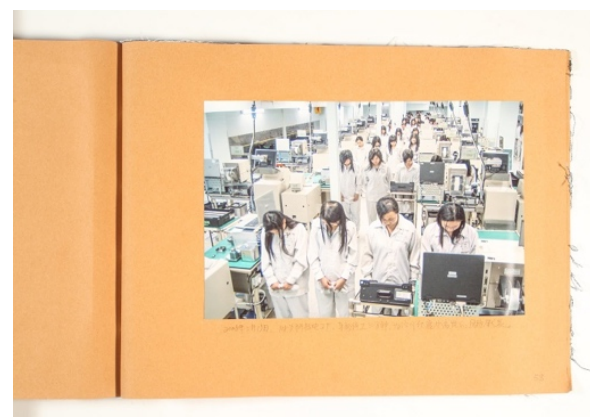

\section{Catalogue 60}

Collection: Lavis Fine Arts

Index No.: 30427_30-60

Title: Shili Technology and Electronics Factory. Work Was Stopped for Three Minutes at Manufacturing Shops to Observe a Moment of Silence for Those Compatriots Who Died in the Wenchuan Earthquake.

Artist: Zhan Youbing

Location: 65QSW21 - 65 Queen Street West, 21 ${ }^{\text {st }}$ floor

Category: Albums

Medium: D2T2 print mounted on kraft paper

Condition: Excellent, minor cockling

Date of Origin: 19 May 2008

Place of Origin: Dongguan, Guangdong, China

Description: None

Size: Image: $27.5 \times 18.3 \mathrm{~cm}(10.83 \times 7.20$ inches $)$

Mount: $42 \times 29.6 \mathrm{~cm}(16.53 \times 11.65$ inches $)$

Signature/Inscriptions: Titled and dated, in pencil, au mount recto

Purchased From: WassinkLundgren (Ruben Lundgren)

Date of Acquisition: 20 October 2016

AMC No.: AMC 16525 


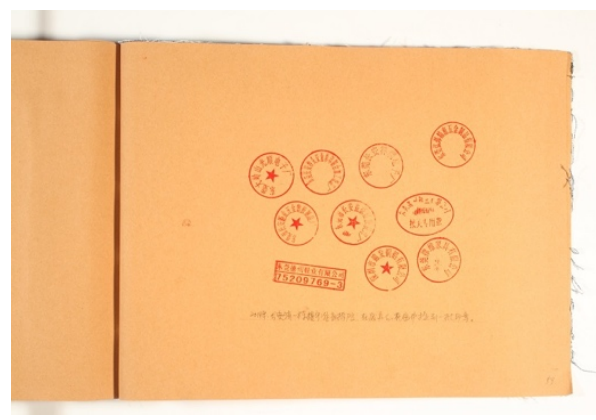

\section{Catalogue 61}

Collection: Lavis Fine Arts

Index No.: 30427_30-61

Title: A Building in Changan Town Is Ready for Demolition. Discarded Stamps Found in Flowerpot.

Artist: Zhan Youbing

Location: 65QSW21 - 65 Queen Street West, 21 ${ }^{\text {st }}$ floor

Category: Albums

Medium: Stamps on kraft paper

Condition: Excellent

Date of Origin: 2014

Place of Origin: Dongguan, Guangdong, China

Description: None

Size: 42 × $29.6 \mathrm{~cm}$ (16.53 x 11.65 inches)

Signature/Inscriptions: Titled and dated, in pencil, au mount recto

Purchased From: WassinkLundgren (Ruben Lundgren)

Date of Acquisition: 20 October 2016

AMC No.: AMC 16525 


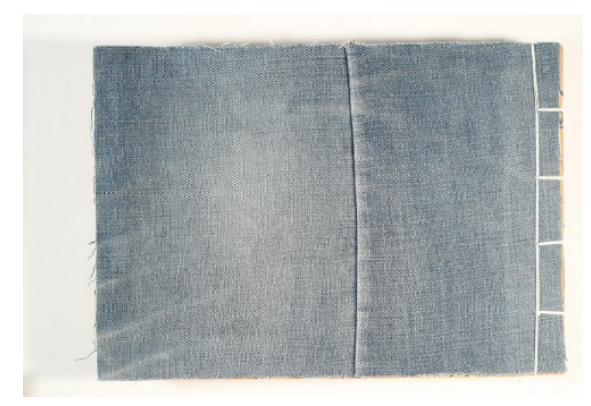

\section{Catalogue 60}

Collection: Lavis Fine Arts

Index No.: 30427_30-v1

Title: Back Cover

Artist: Zhan Youbing

Location: 65QSW21 - 65 Queen Street West, 21 ${ }^{\text {st }}$ floor

Category: Albums

Medium: Denim photo album with embossed title contains 50 D2T2 prints and other objects related to Chinese migrant workers.

Condition: Excellent

Date of Origin: December 2015

Place of Origin: Dongguan, Guangdong, China

Description: None

Size: $42 \times 29.6 \mathrm{~cm}(16.53 \times 11.65$ inches $)$

Signature/Inscriptions: None

Purchased From: WassinkLundgren (Ruben Lundgren)

Date of Acquisition: 20 October 2016

AMC No.: AMC 16525 


\section{Chapter 4. Preservation/Housing Instruction}

\subsection{Preservation Issues}

\subsubsection{Condition of the Album}

The album is in excellent condition at first glance. Benefit from its unique binding style and the hardness of the covers, the album is fairly firm and stable. No significant deterioration or damage is observed. However, cockling has started to appear along the edges of the photographs. According to my preliminary assessment, the cause of this problem may be either the high acidity of the paper $^{46}$ or the unknown adhesive used to adhere photographs to the mounts, since both have direct contact with the images.

\subsubsection{Tests before Preservation}

In order to prepare the best preservation strategy for the album, the first step is to test the acidity of the fiber base to decide whether $\mathrm{pH}$ neutral archival paper should be considered to protect the prints from any acidic material. ${ }^{47}$ It merely requires a simple tool to complete the acidity test, which is a $\mathrm{pH}$ pen. To make the album more permanent, the fiber base must have an alkaline reserve which means a pH of 7.0 or above. Basically, marking the test sample with a $\mathrm{pH}$ pen

${ }^{46}$ Most of kraft paper is manufactured with acidic sulfite so I assume it is acidic. Honghi Tran and Esa Vakkilainnen, "The Kraft Chemical Recovery Process," Tappi.org, 2008, accessed August 02, 2017, https://www.tappi.org/content/events/08kros/manuscripts/1-1.pdf.

${ }^{47}$ According to AMC's policy, no destructive tests is allowed to conduct on any collection, therefore the test sample of the fiber base was provided by photographer Zhan Youbing from Dongguan, China. 
allows the color of the ink to reveal whether the paper is acidic or alkaline. Purple indicates a pH of 7.0 or greater, yellow indicates a pH below 6.5 , which is acidic and not recommended for photographic storage. ${ }^{48}$ Surprisingly, the result of the test showed that the acidity of the fiber base is neutral (alkaline) (see fig.1). Therefore, the cockling problem of the prints is probably the result of the unknown type of adhesive used to adhere the photographs. My hypothesis is that the strength of the adhesive is so strong that it is keeping the photographs from lying flat, a common deterioration issue of photographic albums. ${ }^{49}$

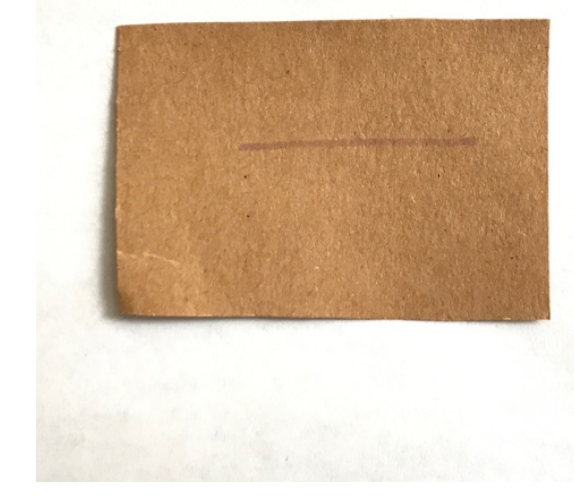

(fig.1) Purple indicates a pH of 7 or above

\footnotetext{
$48 \mathrm{~J}$. Miller and Ellen McCrady, "Comparison of pH Pens on the Market," American Institute for Conservation of Historic and Artistic Works, November 1990, accessed June 21, 2017, http://cool.conservation-us.org/byorg/abbey/ap/ap03/ap03-5/ap03-508.html 49 Shannon Zachary, Conservation of Scrapbooks and Albums: Postprints of the Book and Paper Group/Photographic Materials Group Joint Session at the 27th Annual Meeting of the American Institute for Conservation of Historic and Artistic Works, June 11, 1999, St. Louis, Missouri (Washington, D.C.: Book and Paper Group [and] Photographic Materials Group, American Institute for Conservation of Historic and Artistic Works, 2000).
} 


\subsection{Making of the Box}

Considering the size of the album and the preservation issues discussed above, the most effective housing method for the album is an archival clamshell box, which is constructed with two trays that fold into each other. It not only offers strong and necessary protection for the album against dirt, harmful light and usual wear but also prevents further damage such as exposure to heat and humidity.

Tools and Materials needed:

- Double Walled Cardboard

- Pencil

- Metal Ruler

- Bone Folder

- Blade

- Paper Clamps (2)

- Cutting Mat

- PH Neutral Adhesive (Carr Mclean)

\section{Steps:}

1. I first measured the dimensions of the album: 


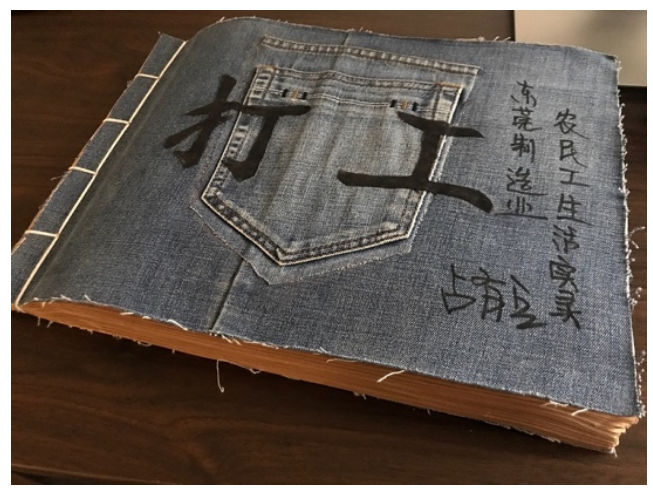

$$
\begin{aligned}
& L-\text { Length of album }=42.5 \mathrm{~cm} \text { (16.7 inches) } \\
& W-\text { Width of album }=30 \mathrm{~cm} \text { (11.8 inches }) \\
& D-\text { Depth of album }=6 \mathrm{~cm} \text { (2.4 inches) }
\end{aligned}
$$

The overall dimensions of the album were $42.5 \times 30 \times 6 \mathrm{~cm}(16.7 \times 11.8 \times 2.4$ inches).

2. I then sized the clamshell box based on following dimensions:

TB - Thickness of board $=0.5 \mathrm{~cm}(0.2$ inches $)$

$L-$ Length of album $=42.5 \mathrm{~cm}$ (16.7 inches)

W - Width of album $=30 \mathrm{~cm}$ (11.8 inches)

D - Depth of album $=6 \mathrm{~cm}(2.4$ inches $)$

The overall dimensions of the box were

$$
\begin{aligned}
& \text { Length }=2 \mathrm{~L}+3 \mathrm{D}+8 \mathrm{~TB} \\
& =2(42.5)+3(6)+8(0.5) \\
& \quad=107 \mathrm{~cm}(42.1 \text { inches }) \\
& \text { Width }=1 \mathrm{~W}+2 \mathrm{D}+5 \mathrm{~TB} \\
& =30+2(6)+5(0.5)
\end{aligned}
$$




$$
=44.5 \mathrm{~cm} \text { (17.5 inches) }
$$

Therefore, a $107 \times 44.5 \mathrm{~cm}$ (42.1 x 17.5 inches) double walled cardboard was needed to complete the box.

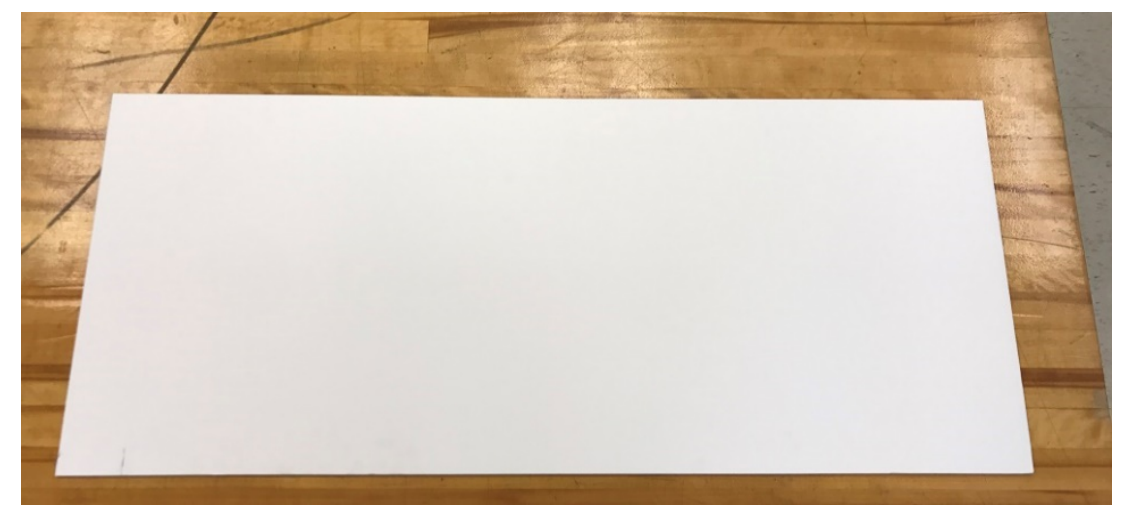

Once the board was ready, I measured and marked the size of the lid and the base of the clamshell box following the instructions below. (Working across the board, horizontally, was the best method for avoiding mistakes.)

3. I measured the length of the lid and the bottom:

$\mathrm{L}$ of lid $=42.5+3 \mathrm{~TB}=44 \mathrm{~cm}$ (17.3 inches)

$L$ of bottom $=42.5+T B=43 \mathrm{~cm}$ (16.9 inches)

a. I first measured the overall length of the lid:

$$
\begin{aligned}
& D+L \text { of } L i d+\text { Spine of the box } \\
= & 6.5 \mathrm{~cm}+44 \mathrm{~cm}+6.5 \mathrm{~cm}(2.6+17.3+2.6 \text { inches })
\end{aligned}
$$

b. Next, I measured the overall length of the bottom:

$$
\text { L of Bottom + D }
$$




$$
=43 \mathrm{~cm}+6.5 \mathrm{~cm}(16.9+2.6 \text { inches })
$$

4. I drew the vertical lines, as following:

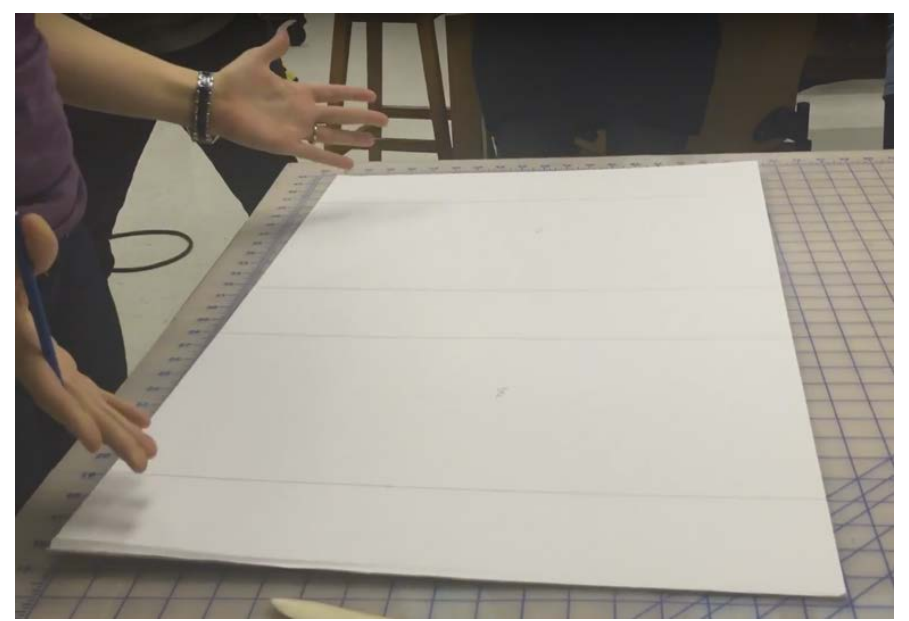

Next, I marked the sides of the box as the Lid and the Bottom to avoid measurement mistakes.

5. I turned the board so the lid was in front:

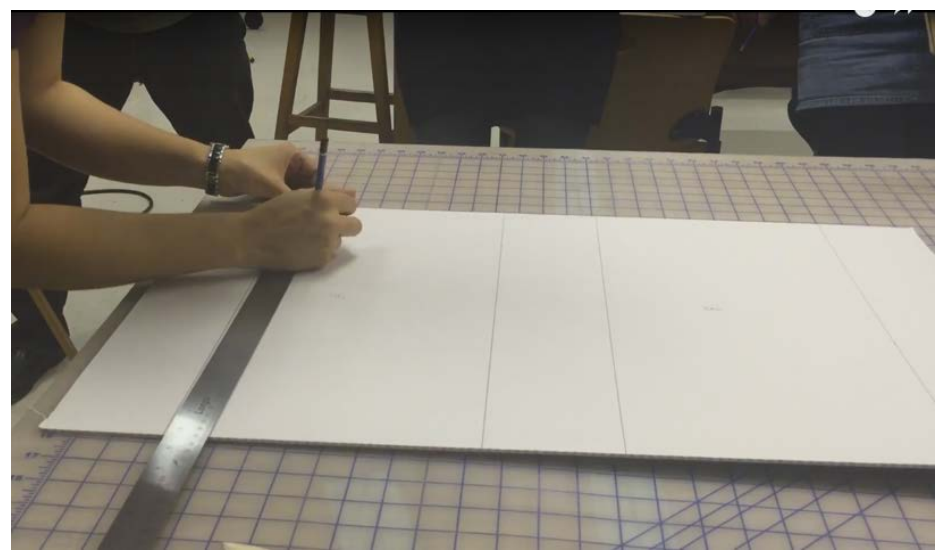

6. Next, I measured the overall width of the lid:

$\mathrm{W}$ of lid $=30+3 \mathrm{~TB}=31.5 \mathrm{~cm}$ (12.2 inches) 


$$
\begin{aligned}
& D+W \text { of lid }+D \\
& =6.5 \mathrm{~cm}+31.5 \mathrm{~cm}+6.5 \mathrm{~cm}(2.6+12.4+2.6 \text { inches })
\end{aligned}
$$

7. I connected the pencil marks together to create the spine:

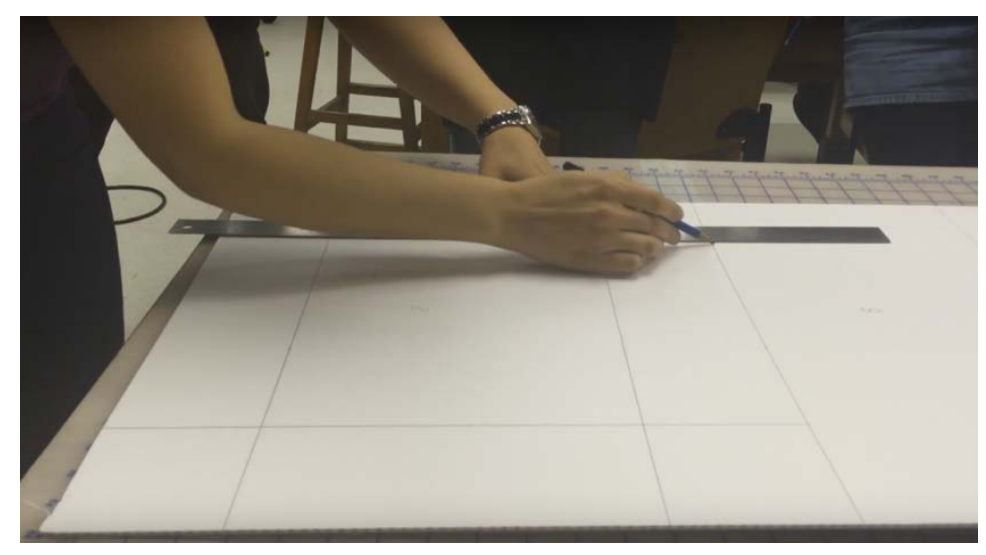

(I reminded myself not to carry the line across the spine because the base was supposed to be smaller than the lid; otherwise, the box would not close.)

8. Once the lid was measured, I turned the board around so the base was in front. The base should be thinner than the lid. I measured $0.5 \mathrm{~cm}(0.2$ inches) (TB) from the spine of the base, as following: 


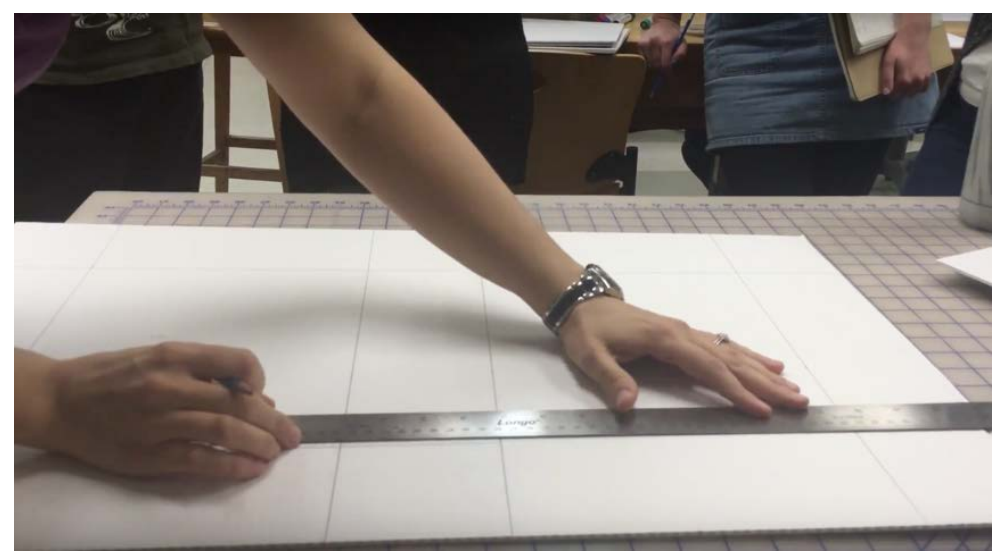

9. After scratching out the box, the next step was to deal with the corners and made sure they fold in nicely. I measured each corner in a thickness of board $(0.5 \mathrm{~cm})(0.2$ inches):

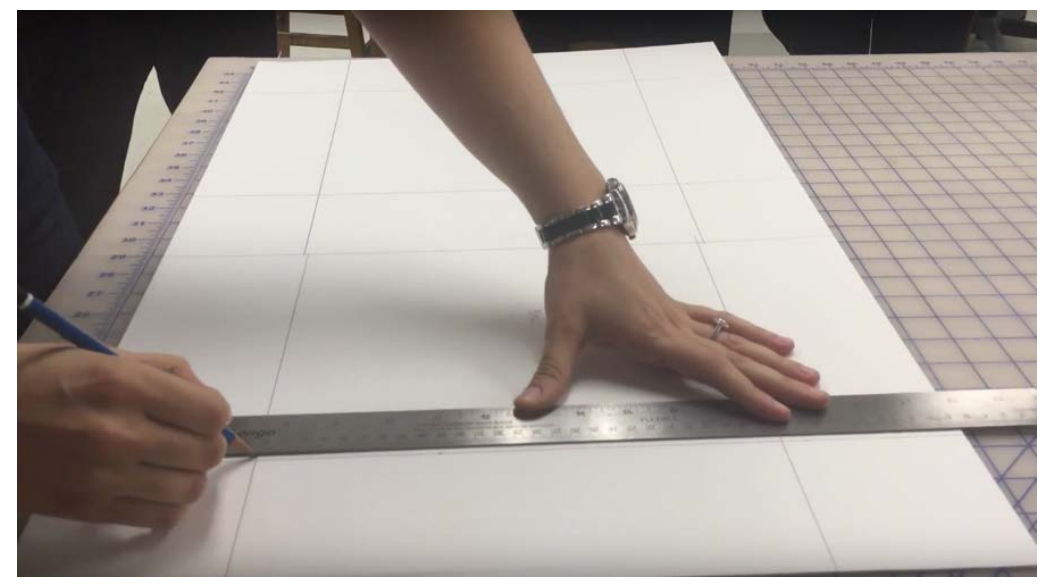

10. Working from the new corner lines, I drew a diagonal line to the corner of the cardboard and labeled the pieces that need to be cut out with an " $\mathrm{X}$ " (including the outer spine pieces): 


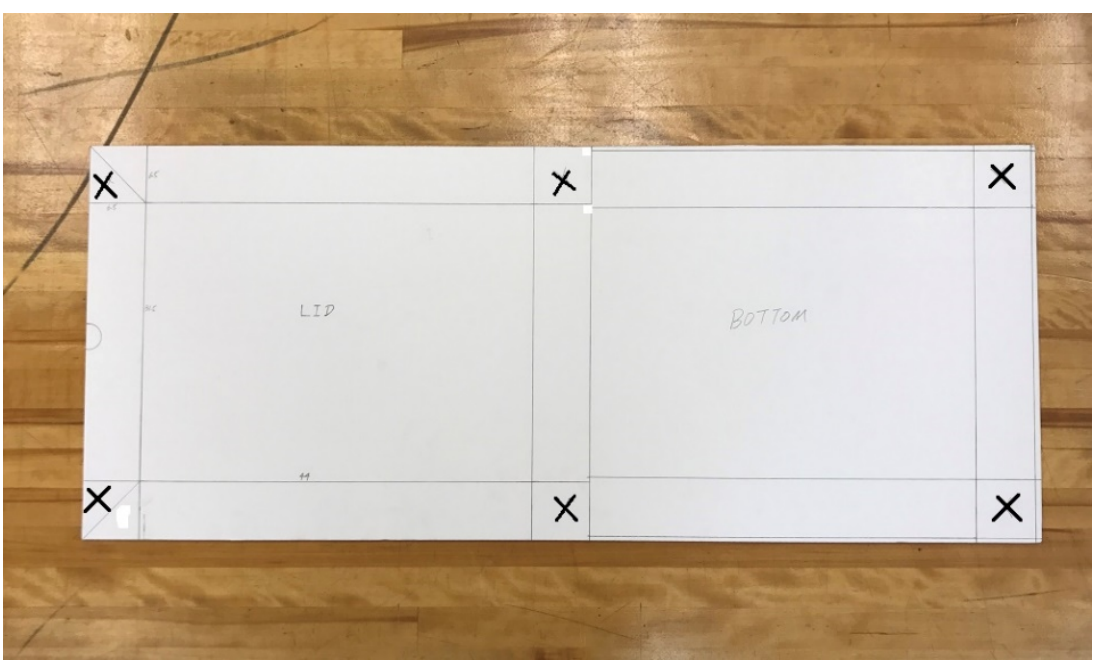

11. I drew a thumb cut in the middle of the edge of the lid, which made it easier to open the box.

12. Next, I scored the new lines that were just created. Then I scored the rest of the lines of the board. (I tried scoring vertically because if scoring sideways, it was hard to get a straight line.)

13. Because the board thickness was reduced in the base in order to make it smaller than the lid (Step 8), in order to make the box close, the board thickness must be decreased from two outer edges of the base. As shown below, I trimmed $0.5 \mathrm{~cm}$ (0.2 inches) from both sides. Then, the base should have the proper shape. 

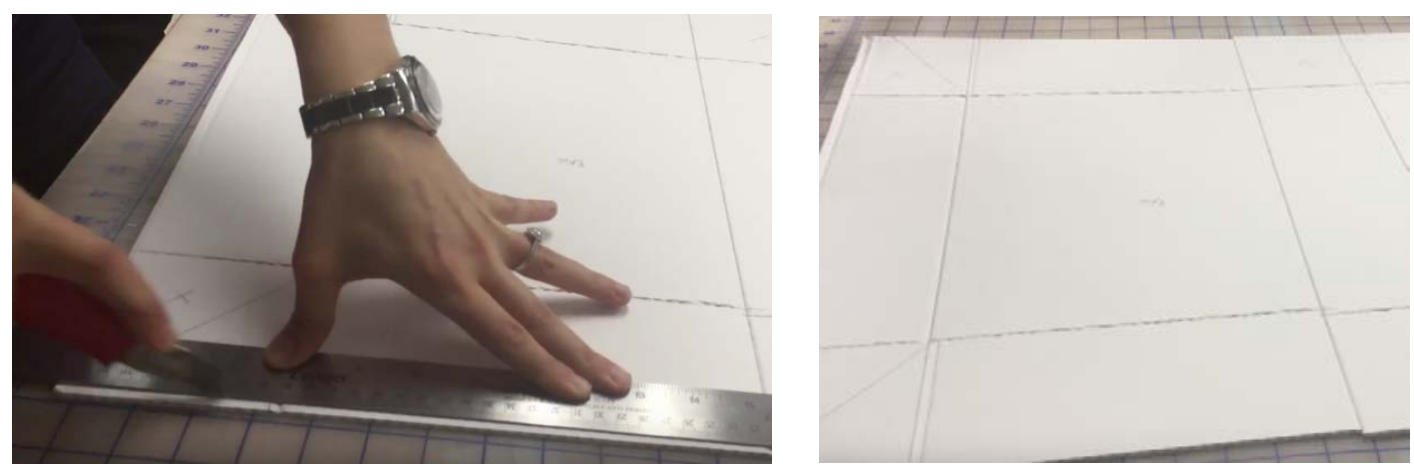

14. Next, I carefully cut out the corners of the lid along with the diagonal line. I started with the edge connecting the width to the side and cut until reaching the corner.

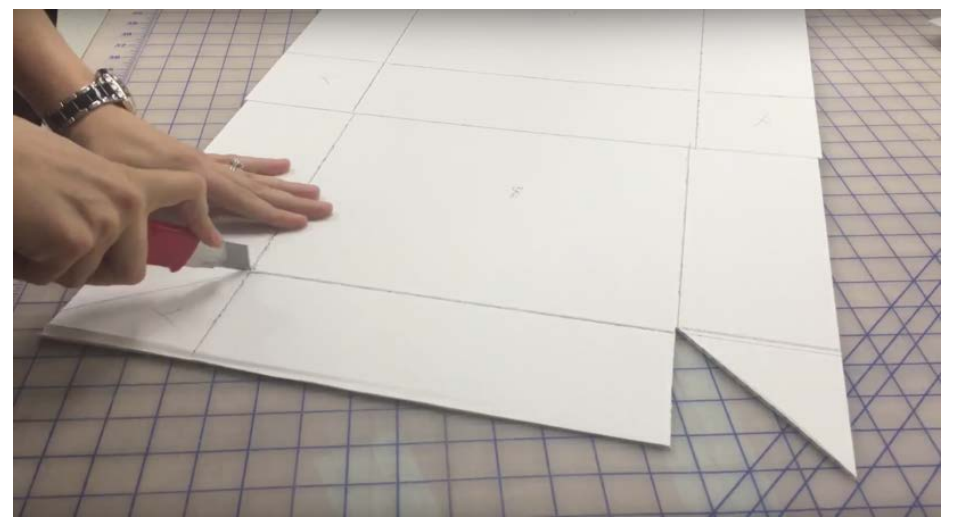

15. When those corners were cut out, I then cut out the inner flaps of the spine and the corners of the bottom, which were the squares marked with an "X". 


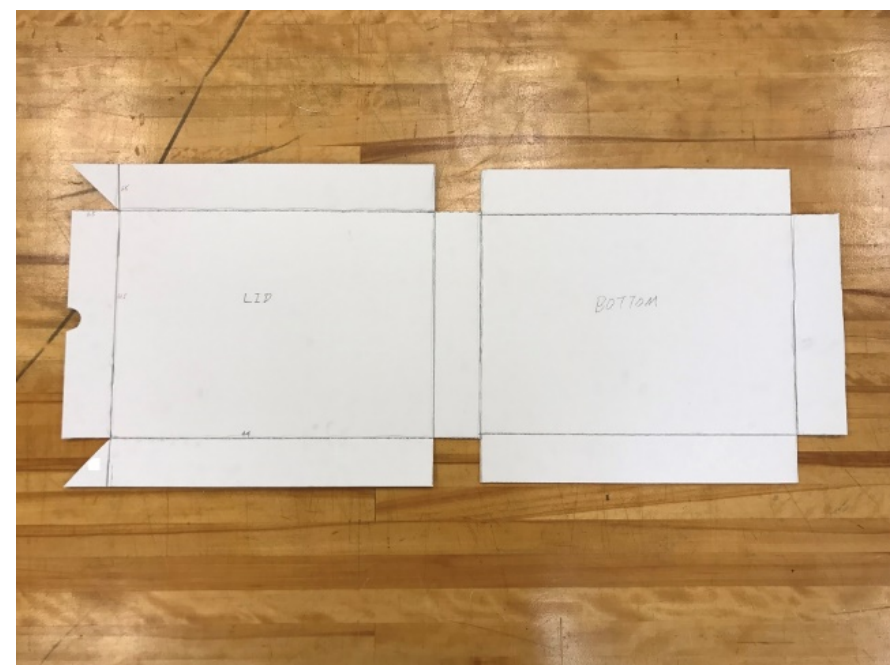

16. I tried prepping all my folds before I glued the box together to make sure everything was in place.

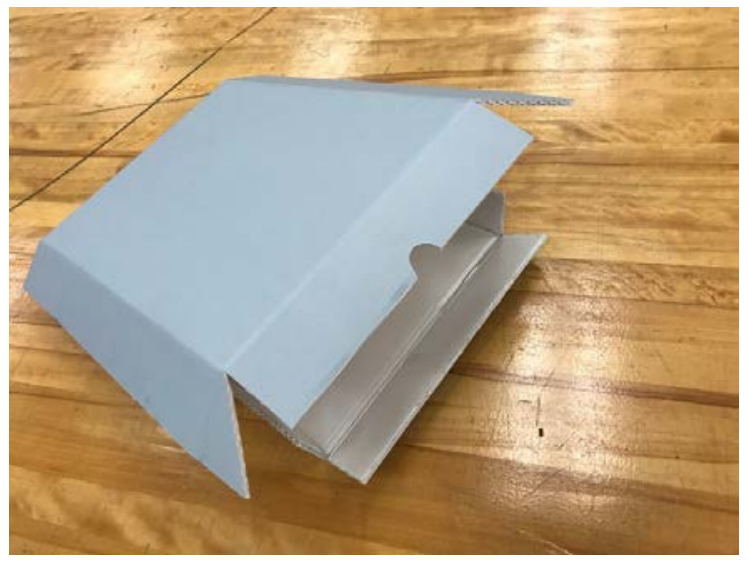

17. Once I made sure everything worked well, I put a small amount of PH neutral adhesive in the middle of the tabs and glued the tabs to the inside width. Next, I used the paper clamps to secure the tabs in position while the glue dried, for 10 minutes. Then, I removed the paper clamps and the box was completed. 


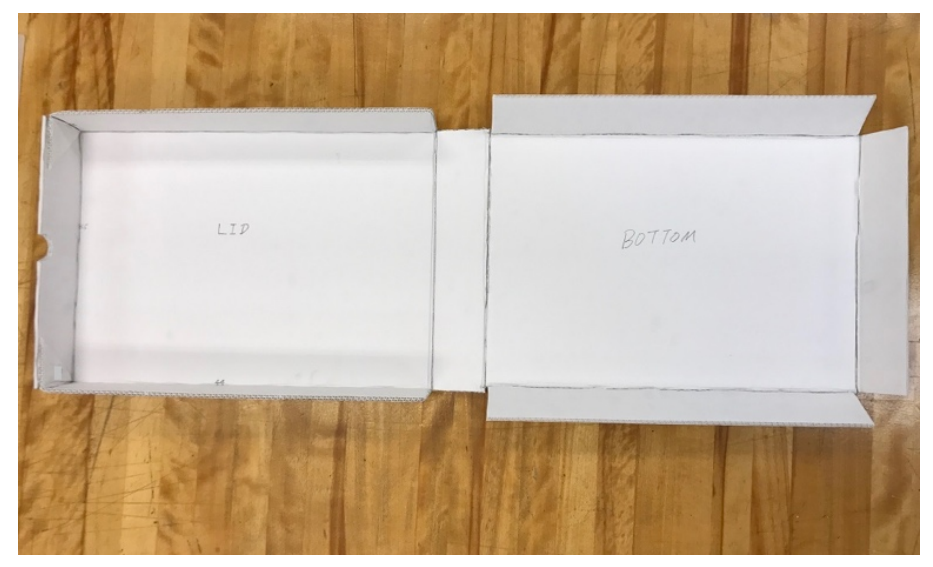

18. I slid the album into the bottom of the box.

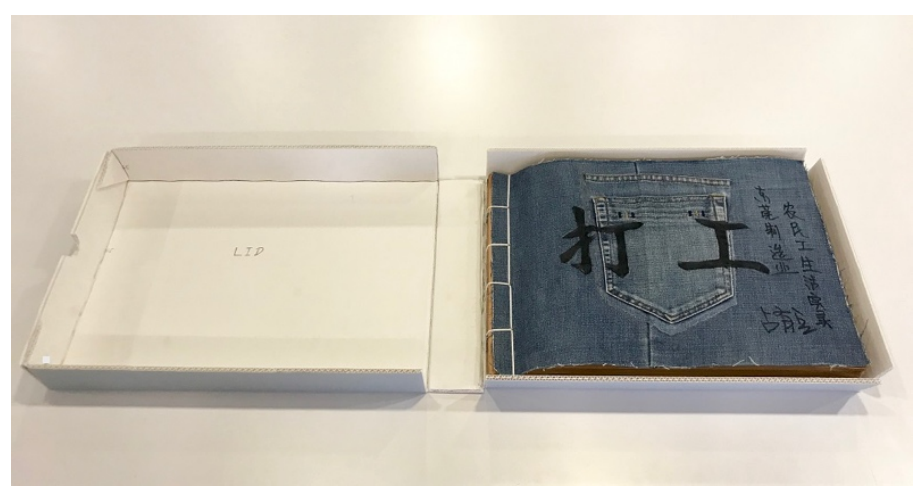

19. Finally, I closed the lid and labeled the album with its index number “LFA30427_30”.

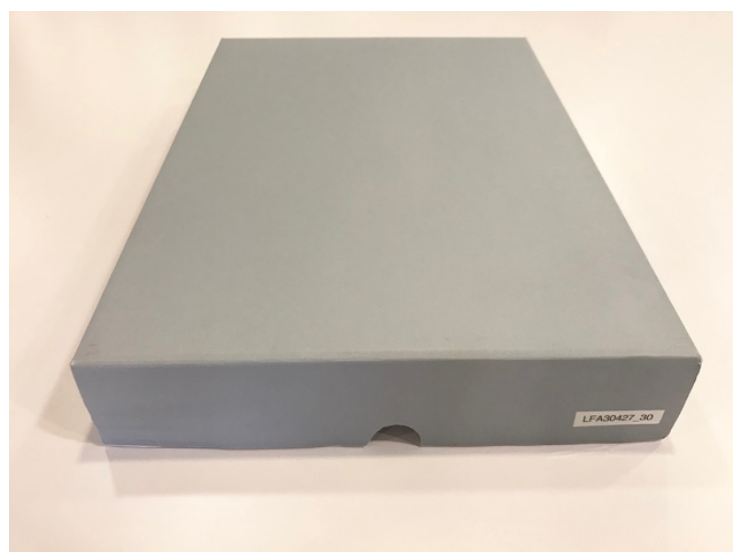




\section{Conclusion}

In my opinion, Work. The Manufacturing Industry in Dongguan: Real-life Stories of Migrant Workers is a unique, well-planned handmade photo album of great social meaning and historical values. With this album, Zhan documents the life of a neglected social group in this particular area, accumulating the collective memory of migrant workers and capturing the spirit of an age. These photographs reflect the situation of migrant workers in China's social transformation, aiming to demonstrate the maker's thoughts about modernization, urbanization, population movements and social equity. It also opens up a window for viewers to pay attention to this social group and reflect on the ongoing process of urbanization.

This applied project has eliminated the language barrier by translating the texts and captions and providing a historical investigation of the social identity of Chinese migrant workers. Cataloguing and digitization of the album made it much easier and convenient for staff and researchers to review the contents of the album and thus avoids unnecessary handling. Last but not least, the preservation issues addressed and the illustrated instructions for the preservation of the album are useful not only for the care of the album but also for future researchers to preserve similar photographic collection. 


\section{Bibliography}

"Agriculture Secretary Han Changbin Talks About Post 1990s Migrant Workers." People.com.cn. February 1, 2010. Accessed April 16, 2017.

http://politics.people.com.cn/GB/1027/10893999.html.

Badger, Gerry. "Ruthless Courtesies: The Making of Martin Parr." In The Pleasures of Good Photographs: Essays. New York: The Aperture Foundation, 2010. Accessed August 02, 2017. http://www.gerrybadger.com/wpcontent/uploads/2012/03/ParrByBadger.pdf.

Carlson, Lage, and Margaret R. Brown. Boxes for the Protection of Books: Their Design and Construction. Washington, D.C.: Library of Congress, 1994.

Chang, Leslie T. Factory Girls: From Village to City in a Changing China. New York, NY: Spiegel \& Grau, 2009.

Chen, Xiaobo. Hou Dengke - Flew Migratory Birds. Beijing: China Renmin University Press, 2007.

Fu, Ping and Youcai Tang. "The Inverted U Shaped Trajectory and the Social Mobility of New Generation Peasant Workers." Zhejiang Social Sciences 25, no. 12 (2009): 41-47.

Gu, Zheng. "Gu Zheng: How Does Photography Bear the Responsibility of the 'Post-Emmanuel Era'." Art.china.cn. September 17, 2014. Accessed April 09, 2017. http://art.china.cn/voice/2014-09/17/content 7238351.htm.

Hao, Meijin. "Analysis of Migrant Workers' Documentary Photography from the Perspective of Visual Culture." Radio and TV Journal 7, no. 5 (2014): 75-77.

He, Guangyi. "Sufferings and Passions, Love and Hate - Documentary Photographer Hou Dengke and Study on His Works." Master's thesis, Northwest University, 2009.

He, Beili and Huang Zeng. Portraits of Chinese Peasants': Story of 900 Million People. Beijing: China Social Science Press, 2004.

Hou, Dengke. Mai Ke. Shanghai: Shanghai Jinxiu Article Press, 2000.

$\mathrm{Hu}$, Xiaohong. "New Generation Migrant Laborers' Dilemma in Self Identity in Social Memory - A Case Study on Many New Generation Migrant Laborers in S County." China Youth Study 20, no. 9 (September 5, 2008): 42-46. 
"Human Capital - Definition of Human Capital in Oxford English Dictionaries." Oxford English Dictionaries. Accessed August 01, 2017.

https://en.oxforddictionaries.com/definition/human_capital.

Jürgens, Martin C. The Digital Print: Identification and Preservation. Los Angeles: Getty Conservation Institute, 2009.

Khemani, R. S. and Shapiro, D. M. "Glossary of Industrial Organisation Economics and Competition Law." Organization for Economic Co-operation and Development. 1993. Accessed April 10, 2017. http://www.oecd.org/regreform/sectors/2376087.pdf.

Li, Yunlei. "Migrant Workers: A Fulcrum of The World. Tang Haowu's Migrant Workers Collection." Photographers of China 15, no. 2 (2008): 80-85.

Liu, Chuanjiang. "China's New Generation of Migrant Workers: Characteristics, Problems and Counter Measures." Population Research 34, no. 2 (2010): 34-56.

Liu, Dingdang. "Zhan Youbing: The Lens Are My Eyes." Siyuefeng.com. October 14, 2014. Accessed May 06, 2017. http://zhanyoubing. siyuefeng.com/article/10047.

Luo, Xia, and Chunguang Wang. "The Causation and Action - Choice of the New Generation of Rural Floating Population." Zhejiang Social Sciences 19, no. 1 (2003): 111-115.

"'Made in China' Opens Tuesday at JCC." The Post-Journal. October 28, 2016. Accessed April 01, 2017. http://content.post-journal.com/?p=702419\%2F-MadeIn-China--Opens-Tuesday-At-JCC.html.

Miller, J. and Ellen McCrady. "Comparison of pH Pens on the Market". American Institute for Conservation of Historic and Artistic Works. November 1990.

Accessed June 21, 2017. http://cool.conservation-

us.org/byorg/abbey/ap/ap03/ap03-5/ap03-508.html

Ouyang, Xingkai. People's road: The Works of Ouyang Xingkai. Beijing: China Minzhu Press, 2012.

Pan, Jiqing, Haiyan Tan, and Na Li. "Rural and Urban Shift of the New Generation of Migrant Workers with an Analysis of Their Employment Experience." Contemporary Youth Research 27, no. 2 (2009): 42-48.

"Preserving and Protecting Photographs: A Buyer's Guide". The American Museum of Photography. 2011. Accessed June 14, 2017. http://www.photographymuseum.com/archival.html\#index. 
Psaila, Denise. "Design and Materials for Photographic Housing." Master's thesis, Ryerson University, 2006.

Sherman, Roger. Market regulation. Boston: Pearson / Addison Wesley, 2008.

Shi, Jinwen. "A Glimpse at the 2016 Taizhou Photography Biennial." Meipian.cn. November 30, 2016. Accessed April 09, 2017. https://www.meipian.cn/9mwery9.

Tang, Haowu. Peasant Workers. Shanghai: Shanghai Jinxiu Article Press, 2011.

Tang, Shouxin. "Changan: Zhan Youbing's Handmade Album was Collected by Martin Parr." Southcn.com. November 24, 2015. Accessed April 10, 2017. http://dg.southcn.com/content/2015-11/24/content 137588899.htm.

Tran, Honghi, and Esa Vakkilainnen. "The Kraft Chemical Recovery Process." Tappi.org. 2008. Accessed August 02, 2017. https://www.tappi.org/content/events/08kros/manuscripts/1-1.pdf.

Wang, Chunguang. "The Social Identity of the New Generation of Migrant Workers and Relationship of Urban and Rural Integration." Sociological Studies 16, no. 3 (2001): 63-76.

Wang, Guohua. "Logical Comparison and Empirical Study on Cultural Adaption of Migrant Workers in Two Generations." Northwest Population Journal 30, no. 5 (2009): 47-50.

Wang, Xingzhou. "On the Group Features of the New Generation of Rural Workers - Taking the Pearl Delta for Example." Journal of Guangxi University for Nationalities 30, no. 4 (2008): 51-56.

WassinkLundgren. "About." WassinkLundgren. Accessed June 21, 2017. http://www.wassinklundgren.com/about/.

Wu, Hongyu, and Guoqiang Xie. "The Characteristics, Interest Claims and RoleChanging of Workers of the New Generation from the Rural: A Survey in Tangxia Town, Dongguan." South China Population 21, no. 2 (April 3, 2006): 21-31.

Wu, Tingting, and Shilin Zhao. "Outsiders' Struggle for Survival - Image of Migrant Workers in News Coverage in Shanghai." Journalism Lovers 24, no. 2 (2009): 10-12.

Xu, Cheng. "China Produces More Than Half of the World's Textile." Xinhua News. February 09, 2016. Accessed August 02, 2017. http://news.xinhuanet.com/fortune/2016-02/09/c 1118017462.htm. 
Yang, Fawei, and Jinyi Yu. "Focusing on the Events with Lens, Provoking Thoughts with Pictures - How to Describe Migrant Workers' Retreat with Photography." Press Outpost 16, no. 4 (2009): 34-35.

Yang, Hu. Chinese Publishing. Cambridge: Cambridge University Press, 2012.

Yang, Juhua. "The Misunderstanding of the Floating Population." Population Research 34, no. 2 (2010): 44-53.

Zachary, Shannon. Conservation of Scrapbooks and Albums: Postprints of the Book and Paper Group/Photographic Materials Group Joint Session at the 27th Annual Meeting of the American Institute for Conservation of Historic and Artistic Works, June 11, 1999, St. Louis, Missouri. Washington, D.C.: Book and Paper Group [and] Photographic Materials Group, American Institute for Conservation of Historic and Artistic Works, 2000.

Zhao, Fang. "'The New Generation'- A Concept Which is Difficult to Be Defined." Sociological Studies 18, no. 6 (2003): 71-83.

Zhao, Shuiping. "The 12th China (Shenzhen) International Cultural Industries Fair." News.timedg.com. May 14, 2016. Accessed April 09, 2017. http://news.timedg.com/2016-05/14/20419747.shtml.

Zucker, Barbara, "Preservation of Scrapbooks and Albums". Preservation Basics: A National Cooperative Information Project, Leaflet no. 1. Washington, D.C.: Library of Congress, 1991. Accessed June 14, 2017. http://www.loc.gov/preservation/care/scrapbk.html. 